\title{
A generalized model for quantifying the impact of Ambient Intelligence on smart workplaces: Applications in manufacturing
}

\author{
Shady Aly a,b,*, Martin Pelikán ${ }^{\mathrm{a}}$ and Ivan Vrana ${ }^{\mathrm{a},{ }^{\text {,** }}}$ \\ ${ }^{a}$ Czech University of Life Sciences in Prague, PEF, Department of Information Engineering, Kamycka 129, 16521 \\ Prague, Czech Republic \\ ${ }^{\mathrm{b}}$ College of Engineering, King Saud University, Kingdom of Saudi Arabia
}

\begin{abstract}
The potentially attractive exploitation of Ambient Intelligence (AmI) seeks improving performance and quality of life of people inside workplaces (e.g., offices, manufacturing work centers, homes). To succeed at making the implementation of AmI fruitful it is important to understand and objectively quantify the logical relationship among the following relevant elements: AmI key enabling technologies, AmI features, basic workplace functions or tasks, and performance measures of the activities of the workplace. Such relationships are fully characterized by ill-structuredness, subjectivity and vagueness. In this article we structure these ill-defined relationships and offer a generalized conceptual model as a foundation for understanding and objectively quantifying such relationships. We then propose fuzzy numbers as an adequate means for expressing the vagueness that is inherent with the subjective nature of the AmI features, technology impacts and characteristics, and relationships with workplace performance measures. The fuzzy numbers are adequately employed through the Analytical Hierarchically Process (AHP) in the form of a Fuzzy-AHP model. We give some example applications from a manufacturing system workplace. The results of the AmI technologies - performance measure assessment frameworks - can be used as a guide in designing smart workplaces and as a valuable insight in adopting the most significant AmI technologies.
\end{abstract}

Keywords: Smart environment/workplaces, Ambient Intelligence (AmI), Multi-criteria Decision Analysis (MCDA), manufacturing systems, Fuzzy Analytical Hierarchical Process (Fuzzy-AHP)

\section{Introduction}

AmI [1] are emerging technologies and concepts that have a high potential for improving the quality of life and performance in various types of environments, especially in workplaces. The prospective improvements that could be gained from applying AmI to the functional performance of people and systems as a whole in the workplace are sought. AmI aims to create an adaptive, friendly and personalized digital environment that understands, learns, reasons and interacts properly and intelligently with user

\footnotetext{
* On leave: Faculty of Engineering at Helwan, Helwan University, Cairo, Egypt.

*** Corresponding author. E-mail: vrana @ pef.czu.cz.
}

needs. This achievement can therefore have positive effects on both the users' and the system's performance.

Some authors have given several definitions to AmI, which have been summarized in Cook et al. (2009) [2]. Cook et al. (2009) [2] and Augusto [3] posed a common definition of AmI: "A digital environment that proactively, but sensibly, supports people in their daily lives." Furthermore, Cook et al. (2009) emphasized the aspects of intelligence and learning in AmI realization. They pointed out that while Ambient Intelligence incorporates aspects of context-aware computing, disappearing computers, and pervasive/ubiquitous computing into its sphere, there is also an important aspect of intelligence in this field. As a result, AmI incorporates artificial intelligence research into its purview, encom- 
passing contributions from machine learning, agentbased software, and robotics. In our article, based on the summarized definitions in [2], we offer an inclusive definition of the AmI environment: the "AmI environment is a system of unobtrusively networked intelligent objects with intelligent and friendly interfaces that can recognize the presence of people and their context and that can learn, adapt and appropriately respond to their needs, habits, gestures, voices, and emotions."

In fact, AmI is considered to be the composition of three emergent technologies: Ubiquitous Computing, Ubiquitous Communication and Intelligent User Interfaces. The aim of the integration of the aforesaid technologies is to make wider the interaction between human beings and information technology equipment through the use of an invisible network of ubiquitous computing devices that compose dynamic computational ecosystems that are capable of satisfying the user's requirements. The realization of AmI concepts will lead to establishing a collaborative working environment, where virtualized entities will communicate to each other. These entities can be humans, artificial agents, web/grid services, virtualized entities representing the real things (not only human beings), or descriptions of human knowledge, among others. These entities will be able to interact with one another in an AmI environment to leverage the full potential of network-centric environments for creativity improvement, boosting innovation, and realizing productivity gains [4]. Such networks will provide the possibility for individuals to experience interaction with human and artificial agents in their working environments. AmI, by means of ubiquitous computing and communication technologies, is anticipated to have a large amount of impact on future technologies, business paradigms and systems, especially in manufacturing and decision support systems. Because of this reality, there is currently a strong emerging desire of researchers and practitioners to investigate the impact of implementing AmI on the performance of systems and people in various types of environment. This desire motivates researchers to study and characterize the relationships among the AmI enabling technologies, the characteristics of the AmI environment and the performance of the systems and people living and working within such an AmI environment.

A considerable amount of research has been conducted in the field of AmI. Mostly, the literature focuses on certain aspects of AmI, on how an ambient intelligent environment and frameworks could be created, the key technologies that enable AmI, the subjective evaluation of AmI advantages and the role of Artificial Intelligence in providing and implementing AmI. The recent research record extensive relies on intelligent multi-agent systems. For example, Serrano \& Botia in 2010 [5] introduced a new methodology that was based on the use of Multi-Agent-Based Simulations (MABS) for the testing and validation of AmI-based Ubiquitous Computing (UbiCom) systems. They pointed out that the motivation for this methodology is its application to UbiCom large-scale systems, in which large numbers of users are involved in applications that address dangerous environments. Hu et al., in 2011 [6], proposed a multi-agent framework to support applications development of ambient systems. They stated that in the programming model of the proposed framework, ambient systems can be developed by collaborations between mobile agents and service (or resident) agents, where resident agents provide application services on devices and mobile agents provide communications services on behalf of the owner applications. Tapia et al. in 2011 [7] presented the integration of the Hardware-Embedded Reactive Agents (HERA) Platform into the Flexible and User Services Oriented Multi-agent Architecture, called (FUSION@), which is a multi-agent architecture for developing AmI systems; this architecture integrates intelligent agents with a service-oriented architecture approach. Tapia et al. stated that because of this integration, FUSION@ has the ability to manage both software and hardware agents using self-adaptable heterogeneous wireless sensor networks. Santofimia et al. in 2011 [8] proposed a treatment of two essential aspects of systems for ambience intelligence: event management and response generation, through the use of an ambient intelligence semantic model that describes what actions and events are taking place, how they are connected and how computational systems should think about their meaning. Bosse et al. in 2012 [9] presented the development of a toolbox that can be used by a modeler to design Ambient Intelligence applications. This toolbox contains a number of modelbased reasoning methods and approaches to control reasoning in the AmI environment. Dipsis \& Stathis in 2012 [10] pointed out that the cost of state-of-the-art devices and robots is critical for the uptake of Ambient Intelligence (AmI) and that one way to use lowcost hardware for both devices and robots is to run smart software remotely as agents deployed on computationally rich environments. They considered that the devices and the robots can be seen as the avatars 
of agents, while the way that devices and agents are related might be considered to be an Ambient Ecology.

Other researchers were devoted to experimentation on the realization of AmI environments, including the work conducted by [11] to promote multimodal interactions. They presented a novel approach based on model-driven development to seamlessly integrate textual information and knowledge resources and to automatically and effortlessly adapt question-answering systems to be useful for restricted-domain AmI environments, such as e-Science. Other related work by De Paola et al. in 2012 [12] described the implementation of a test-bed that provided the hardware and software tools for the development and management of AmI applications based on wireless sensor and actuator networks, with the main goal being energy saving for global sustainability. They presented a sample application that addresses temperature control in a work environment through a multi-objective fuzzy controller, while accounting for users' preferences and energy consumption.

Relevant to this research in the field of manufacturing, the AmI concepts and technologies have been applied to several areas, such as business, engineering, and industry. Relevant to this research, Kovács et al. in 2006 [13] and Kopácsi et al. in 2007 [14] introduced some basic research results that were achieved in the application of Ambient Intelligence for improving Product Life-cycle Management for manufacturing companies and service engineering for serviceoriented companies. Maurtua et al. in 2006 [15] presented the research activities of analyzing technological, human and social needs and implications for AmI in the manufacturing field.

With respect to the uncertainty and the use of fuzzy logic, which is one of the tools proposed in our research (and more deeply in attempting to address the uncertainty and subjectivity that is associated with understanding relationships between AmI features and the AmI technologies in the workplace environment), some research that involves the utilization of fuzzy logic has been conducted. Doctor et al. in 2005 [16] presented a novel approach for realizing the vision of ambient intelligence in ubiquitous computing environments (UCEs). This approach is based on embedding type-2 fuzzy intelligent agents in UCEs. These agents can handle the different sources of uncertainty and imprecision in UCEs, to obtain a good response. Acampora \& Loia in 2008 [17] proposed a ubiquitous fuzzy computing method for Ambient Intelligence, to address the reverse interactions, from devices to users, in contrast to the prior attention on the interaction from users to devices.

An extensive study of the literature has revealed that nearly no research has been performed that focuses on the influence of AmI on the performance of manufacturing systems or, in general, workplace environments. A large step has been achieved toward assessing the influence of AmI on managerial decision support in the workplace through the research conducted in [18], in which conceptual models were developed for modeling the relationships among AmI enabling technology, AmI enabled features and the performance measures of managerial decision support (MDS). The developed models constitute the basic framework for further development in this respect within this research. Indeed, also very few or even no research attempts have focused on developing formal and objective models to quantify the relationship between AmI attributes and the performance of people and systems. Research attempts to achieve a deeper move toward being able to assess the impact of the ambient intelligence tools and technologies on the performance of manufacturing system functions that were built in the work were conducted by Aly \& Vrana in 2011 [19], where an objective assessment model was presented to quantify the influence of the AmI features on the managerial decision support workplace.

This article presents a generalized model for the quantification of the impacts of AmI features and attributes on the performance of any workplace. The model hierarchically structures the relationships between AmI technologies and the workplace performance measures. However, because the relationships of the impacts of AmI technologies on the workplace performance measures and their significances are mainly subjective or qualitative, and so are the AmI features, which are also subjective or qualitative concepts (e.g., adaptability, context awareness, and friendliness). Because human judgment is viewed as uncertain, vague, or inexact, one way is to resort to human experts to linguistically (qualitatively) quantify and assess their values using a psychometric numerical scale. One common and efficient method to manipulate and quantify the inexactness or vagueness due to subjectivity is to employ fuzzy sets (i.e., to express individual variables as fuzzy quantities). Fuzzy-AHP has impactful advantages, which are the capability to consider a non-homogenous mix of quantitative and qualitative (subjective) factors and concepts and the capability to account for the vagueness that is associated with quantifying intangible or subjective concepts. The 
proposed model effectively enables handling a wide range of subjective and vague concepts that are hierarchically embedded in the subtle relationship between AmI features and the performance measures of the workplace. Thus, one can understand the various impacts of every AmI technology and feature on the workplace performance, which consequently could be utilized more wisely and efficiently. These goals will be achieved through a Hierarchical fuzzy-AHP model that adequately fits the given decision-making problem through modeling the hierarchical relationship. It permits manipulating many subjective and vague concepts through the use of an AHP pair-wise comparison of fuzzy values that represent the significance of the AmI technologies and the features of the functionalities and performance measures. The article structures such a relationship and proposes a Fuzzy Analytical Hierarchical Process (Fuzzy-AHP) model for measuring the vague influence of AmI on the manufacturing system performance measures, as a typical example workplace application that still has not been investigated.

This article is organized as follows. Section 1 introduces the main interest of this article in addition to the results of a recently conducted relevant literature review. Section 2 reviews the key AmI attributes and technologies. In Section 3, the impact of the proposed AmI generalized hierarchical model on the workplace performance is introduced. Section 4 presents the Fuzzy-AHP model for quantitatively assessing the impact of AmI technologies on the performance of the workplace. An illustration case study example is provided to demonstrate the effectiveness of the proposed model at objectively mapping the influence of the AmI technologies on the workplace performance through a hierarchical relationship and FuzzyAHP in Section 5. Lastly, a conclusion is stated in Section 6.

In the next section, the basic AmI features and key enabling technologies are highlighted.

\section{AmI attributes and key enabling technologies}

The objective of AmI is to broaden the interaction between human beings and digital information technology through the use of ubiquitous computing devices. Conventional computing primarily involves user interfaces (UIs), such as a keyboard, mouse, and visual display unit, while a large ambient space that encompasses the user is not utilized as much as it could be. In this section, we review the first component of the vague relationship between AmI technologies and workplace performance measures, which are the distinct AmI attributes or features that have been identified and are summarized in [18].

The term AmI was defined by the Advisory Group to the European Community's Information Society Technology Program STAG in 2005 [1], as "the convergence of ubiquitous computing, ubiquitous communication, and interfaces adapting to the user" [21]. Ubiquity involves the idea that something exists or is everywhere at the same time on a constant level, for example, hundreds of sensors placed throughout a household or in a factory where some number of agents are combined into the network, while the network can monitor the operation of household equipment, machine tools or the production of any future product. AmI incorporates properties of distributed interactivity (e.g., multiple interactive devices, remote interaction capabilities), ubiquitous computing (the "invisible" computer concept), and nomadic or mobile computing. AmI can provide the user with a virtual space that enables flexible and natural communication with the computing environment or with other users, providing input and perceiving feedback by using proportionally all of the available senses and communication channels. AmI, on the other hand, uses this space, e.g., in the form of shape, movement, scent and sound recognition. This information media became possible through new types of interfaces and will allow a drastically simplified and more intuitive use of devices. Wireless networks will be the dominant technology for communication between these devices. The combination of simplified use and its ability to communicate will eventually result in increased efficiency for users and will create value, leading to a higher degree of ubiquity for the computing devices. Examples of such devices range from common items such as pens, watches and household appliances to sophisticated computers and production equipment. Therefore, AmI features are avatars of utilization of AmI key enabling technologies. The AmI key technologies can be summarized into 6 different complementary technologies: sensors, smart actuators, wireless networks, securities/privacy technologies and human computer interaction (HCI) technologies, as depicted in Fig. 1.

The review of AmI technologies and their relationships to AmI attributes has been presented in [20]. The subjective study of the conducted literature and Inter- 


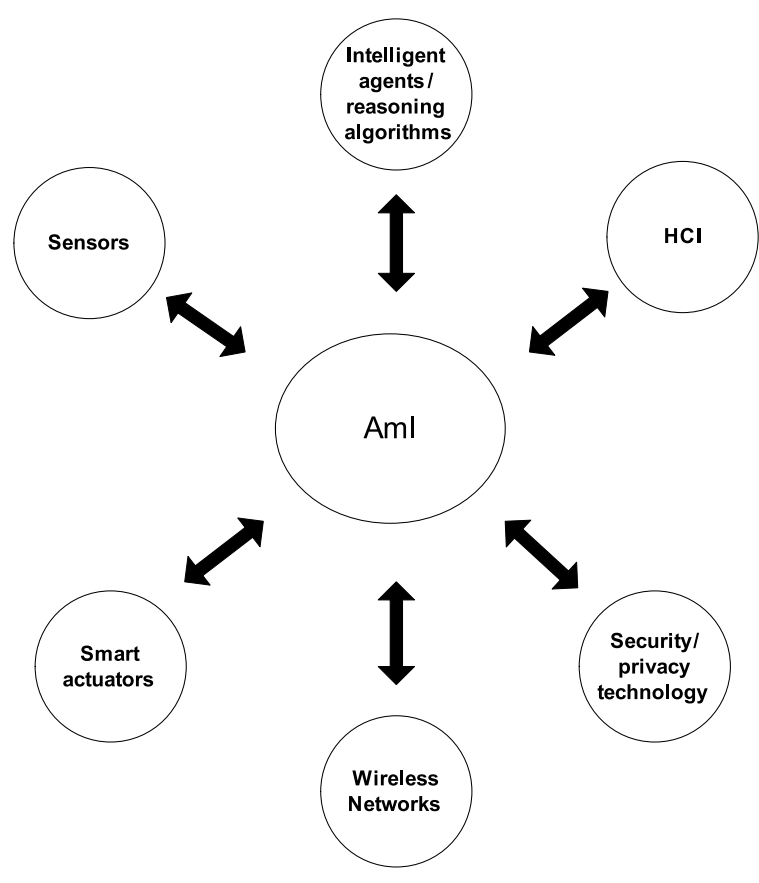

Fig. 1. The Key AmI enabling technologies.

net resources survey [20-22] has clearly identified several distinct features of AmI, as follows (see Fig. 2):

- Proactive response: this type of response can be accomplished through learning users' behaviors and preferences when interacting with the system and anticipating future moves and desires.

- Adaptive response: this type of response involves the perception of the system as intelligent by people who naturally interact with the system, while the system automatically adapts to their preferences.

- Context-awareness: context-awareness provides computing environments that have the ability to usefully adapt to the services or information that they provide. It is the ability to implicitly sense the users' needs and automatically derive them. Context-aware applications are more attentive, responsive and aware of their users' identity and state and their users' environment.

- User friendly interfaces: user interfaces should exhibit simplicity of learning, communication and intuitive usability by relatively low-skills or general-skilled users.

- Handling different sources of uncertainty and imprecision: application systems should be designed to address diverse sources of vagueness, uncertainty, and imprecision; this type of source frequently occurs in today's real world.

- Unobtrusive intelligence: applications systems should exhibit implicit, flexible, seamless and natural intelligence to users.

- Ubiquitous computing: this type of computing is accomplished through the presence of pleasant computing devices everywhere in the system.

- Ubiquitous communication: access to network and computing facilities everywhere.

- Multimodal and broad interaction: broad interaction through enabling: shape, movement, scent and sound recognition and outputs between human beings and digital information technology.

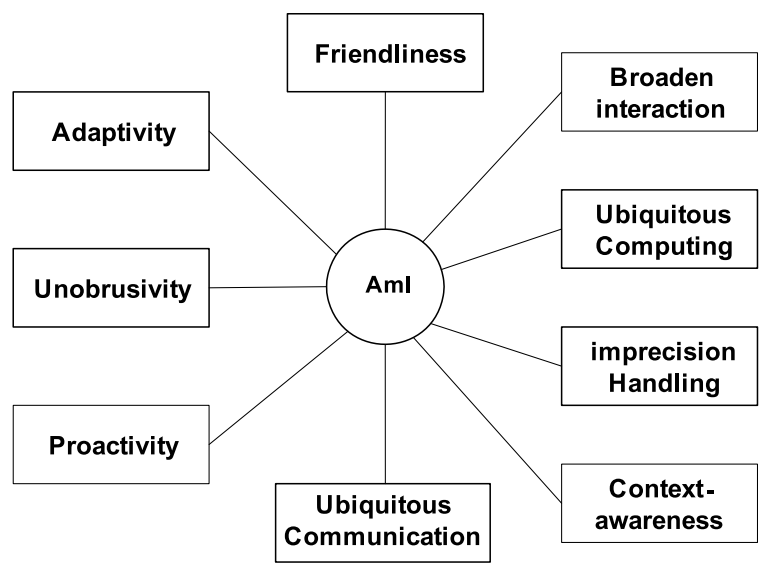

Fig. 2. The nine main features of AmI.

An extensive review and classification of AmI applications, technologies and research can be found in (Hong et al. (2008), Augusto (2008)) [20,21].

The next section presents the generalized hierarchical conceptual model of AmI's impact on workplace performance.

\section{The generalized hierarchical model of AmI impacts on the workplace performance}

The key appealing aspect of an AmI implementation is to improve the performance and conditions in the workplace. This aspect could be more effectively achieved through understanding and structuring the relationships between AmI technologies and the performance measures of a workplace; this aspect is still an open question in research studies.

We can imagine that a workplace is composed of several subsystems, each of which has a specific set 


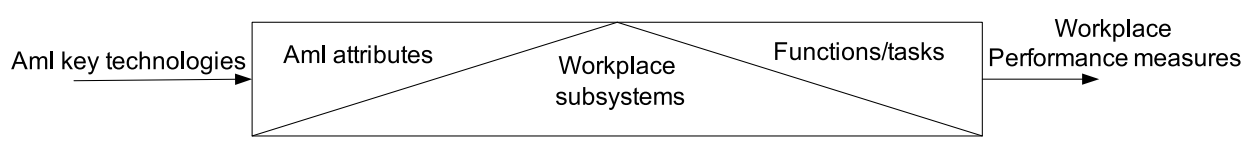

Fig. 3. Elements of the AmI impact on the workplace relationship.

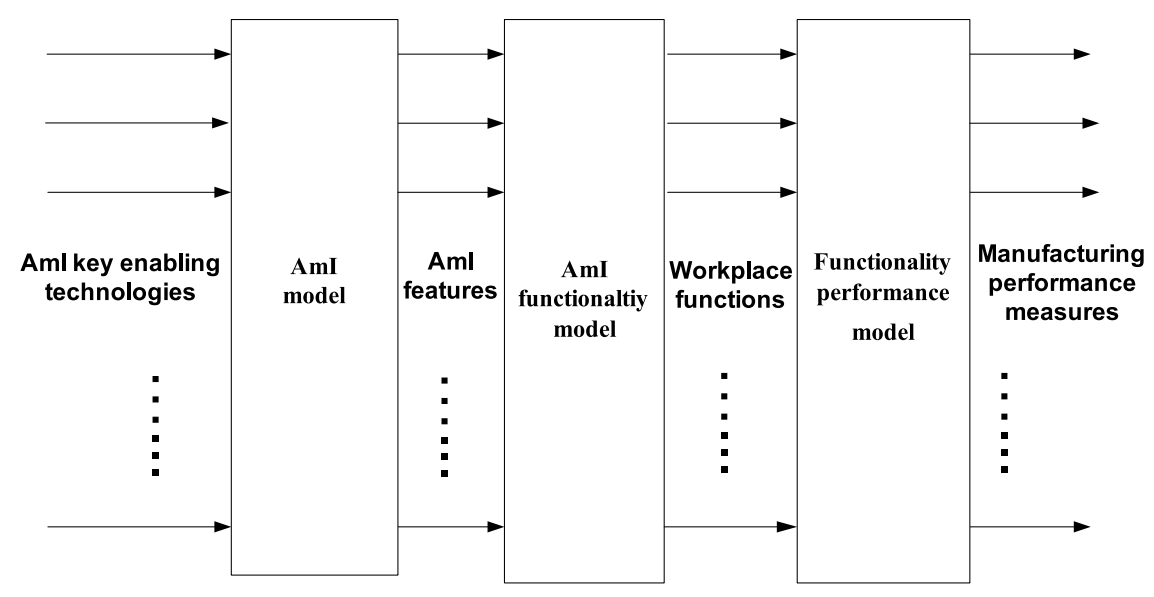

Fig. 4. The conceptual model of AmI impact on the workplace.

of functions, and those functions could be, in turn, divided into tasks and further divided into subtasks. Figure 3 describes briefly this set of conceptual relationships. In Fig. 3, upon implementation of AmI technologies, the realization of AmI attributes or features (adaptivity, context awareness, multimodality, among others) becomes possible, which individually or cooperatively affects the function and task performances of the workplace subsystems and results in the whole system performance outcome.

In fact, the first attempt to develop an objective relationship among AmI features from one side to the performance of the workplace has been performed in Aly \& Vrana (2009) [18]. This research was conducted within the project Ambient Intelligence for Managerial Decision Support (AMIMADES) (2006-2009). In Aly \& Vrana (2011) [17], we had extended the conceptual AmI models proposed in Aly \& Vrana (2009) [16] into an objective model through constructing a hierarchical model to structure such inherently subjective and vague relationships and through employing fuzzy sets. In this study, we intend to further extend and generalize the studied relationship to be useful for applications in various types of workplaces.

The relationship among AmI key enabling technologies and the workplace measures of performance can be logically structured as composed of the three-stage sub-relationships among three groups, which are the following:
- The mapping (i.e., the significance relationship) from AmI key enabling technologies into AmI attributes/features.

- The significance relationship between AmI attributes/features and the workplace key performance functions.

- The significance or importance relationship between the workplace's key functions and its performance measures.

The three stage conceptual relationships model is depicted in Fig. 4. One important challenging problem with the model shown in Fig. 4 is that it comprises mainly subjective (i.e., qualitative) aspects, which exert inherent vagueness on the quantification and objectivity of the relationships and accurate computation of their outputs. However, still the proposed model contributes to simplifying the main AmI technology performance relationships through hierarchically structuring them. The justification and effective implementation of AmI technologies should be based on an objective quantification of their impact on the subject system or the workplace. To be able to effectively and beneficially employ the AmI technologies on improving the performance and quality of such a workplace, it is necessary to understand the impact of those technologies in realizing the AmI features and positively affecting the performance of the functional components or subsystems at the workplace. This task cannot 


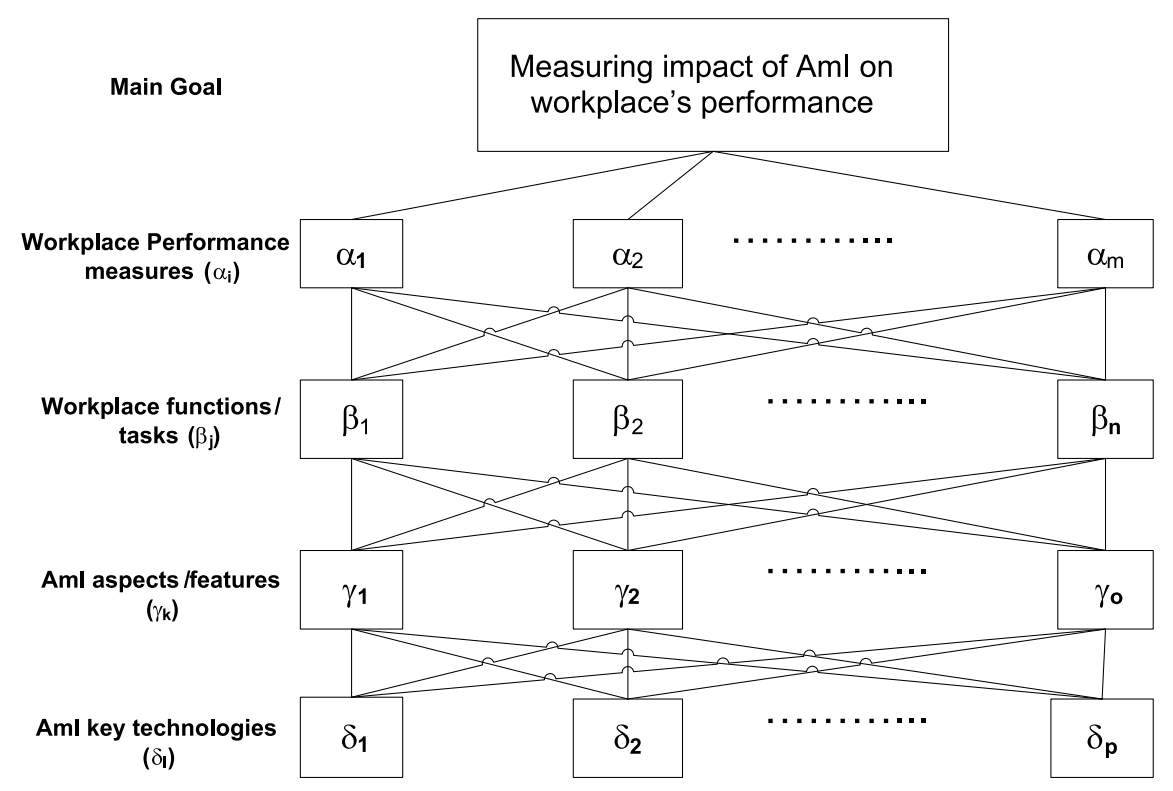

Fig. 5. The generalized hierarchical model of assessing AmI impact on the workplace.

be accomplished easily directly, but it might be accomplished by restructuring the relationships into their logical intermediates. Such restructuring could be simpler because the complexity in understanding and measuring the relationship will strongly decrease when broken down into smaller/partial relationships.

To objectively assess the impact of AmI on the workplace performance and given the hierarchical nature of the relationship and multitudes of AmI technologies, AmI features, workplace functions, and workplace performance measures, the model in Fig. 4 can now be more objectively and conveniently depicted as in Fig. 5. Figure 5 describes an assessment decision-making problem of AmI impact on the workplace performance. It consists of layers, each of which comprises one of four elements of the studied AmI impact relationship. The goal of the problem is placed at the top, followed by the elements of the relationship. The depicted hierarchy is now adequate for implementing several hierarchical decision-making tools and, among them, the most common and widely used tool, the Analytical Hierarchy Process (AHP) [23], which will be utilized along with fuzzy logic in a Fuzzy-AHP model to assess the impact of AmI on the performance in the workplace.

The top of the above hierarchical tree is the main goal, which is to measure the impact of the AmI on the workplace performance. This goal is composed of the following conceptual levels:
- Level $\alpha$ : the performance measures $\left(\alpha_{\mathrm{i}}\right)$ on the functions performed in the workplace; $\mathrm{m}$ is the total number of measures

- Level $\beta$ : the key functions $\left(\beta_{\mathrm{j}}\right)$ performed in the workplace, $\mathrm{n}$ is the total number of functions

- Level $\gamma$ : the AmI features or aspects $\left(\gamma_{\mathrm{k}}\right)$; o is the total number of AmI features

- Level $\delta$ : the AmI key enabling technologies $\left(\delta_{1}\right)$; $\mathrm{p}$ is the total number of assessed AmI technologies

In this research, we propose a Fuzzy-AHP [23] model as an adequate tool for handling the proposed hierarchical structure; this tool is full of subjective concepts that could be quantified only using a linguistic description and psychometric numerical scale based on a human expert's judgment. Fuzzy logic is an effective technique for handling vague and inexact quantitative and qualitative assessments that lead to vague concepts and relationships, and therefore, the proposed FuzzyAHP model is highly suited to tackling the hierarchical structure and the vague subjective concepts simultaneously.

Fuzzy logic theory is not a goal of this study; instead, it serves as a well-proven formal theoretical method for expressing ambiguity, which is also inherent in quantifying the ambient intelligence impact on smart workplaces. To help readers who are not familiar with fuzzy set theory, we have added Appendix B for a brief introduction to fuzzy sets and fuzzy num- 
bers and their relevant operations. The purpose of Appendix $\mathrm{B}$ is to improve the understanding of the role of fuzzy numbers in expressing the uncertainties that are involved in assessing subjective, vague concepts and relationships. Appendix B will also help in understanding the Fuzzy-AHP procedure mathematics that is used in this article.

The next section reviews and introduces FuzzyAHP, which will be used to quantify the vague concept of the hierarchical relationship model.

\section{A Fuzzy-AHP model for measuring the impact of AmI on the manufacturing workplace's performance}

In this article, fuzzy numbers will be used to quantify vague or inexact experts' judgments on the significance relationships among the elements of the four levels of the proposed assessment framework: AmI technologies, AmI features, workplace functions and workplace performance measures.

AHP was used in [22] for evaluating decision support systems under multi-criteria goals. Fuzzy-AHP extends Saaty's AHP by employing fuzzy logic in its mathematical procedure. In 2008, Aly and Vrana [23] developed a fuzzy version of Saaty's AHP method.

In this section, we present an application of a developed Fuzzy-AHP for modeling the vague and complex relationships among AmI technologies and workplace performance criteria. As Fig. 5 indicates, the proposed Fuzzy-AHP hierarchy will include 4 levels. The top level (level 0) is the goal of the hierarchy, which is assessing the influence of AmI on the workplace performance. Then, the next level (level $\alpha$ ) is the major assessment criteria; those criteria are the workplace performance measures aforementioned in this article. The second level (level $\beta$ ) contains the main functions or tasks that are performed in the workplace. The third level (level $\gamma$ ) contains the AmI features and, lastly, the lowest level (level $\delta$ ) includes the AmI key enabling technologies.

The basic notion behind the arrangement of the above Fuzzy-AHP hierarchy, which constitutes one additional achievement of this article, is that the positioning of the AmI technology level as the lowest level in the hierarchy will lead to and make it possible to compute their overall priorities or significance with respect to the workplace performance measures. The consecutive evaluation of priorities via the intermediate levels (i.e., the AmI features and workplace functions) and the utilization of the simple pair-wise expert comparisons using fuzzy numbers or alternatively fuzzy linguistics highly contributes to minimizing the assessment complexity caused by the subjectivities that are inherent in the constituents of the four levels of the hierarchy. This relationship will be illustrated more formally later in this article. In the next sub-section, we review the computational procedure of Fuzzy-AHP developed in [23] and state how it could be utilized by the problem addressed in this research.

\subsection{The Fuzzy-AHP procedure}

Aly \& Vrana in 2008 [23] proposed and successfully utilized a fuzzy version of Saaty's AHP method that can be considered to be a fusion of the most common Fuzzy-AHP procedures that were recently developed and agreed upon. In this article, Fuzzy-AHP can be utilized to assess the influence of AmI on the workplace performance. The following text explains how the Fuzzy-AHP procedure can be computationally applied to the problem being addressed.

In Fuzzy-AHP, fuzzy numbers are used with pairwise comparisons to compute the preference rating of each compared pair of concepts, function or criteria that are included in the Fuzzy-AHP hierarchy in Fig. 5. Thus, all of the elements in the judgment matrices and weight vectors are represented by fuzzy numbers.

Using fuzzy numbers, the expert decision-maker can evaluate the relative importance of various elements with the three levels of the Fuzzy-AHP hierarchy in Fig. 5, which pertaining to workplace performance measures (level 1), decision support functions (level 2), and AmI features (level 3). Thus, fuzzy judgment matrices are built using fuzzy numbers rather than crisp numbers, as was the case in AHP [24]. The Fuzzy-AHP procedure is as follows:

- Step 1: In a fuzzy environment, the crisp judgment scale used in comparing the relative importance of the compared factors should be first converted into the fuzzy judgment scale, which is made of fuzzy numbers. This scale, as given in Table 1, has been proposed and described in [25], and was also recently utilized in several research studies on the application of fuzzy-AHP, including [29]. The scale simply transforms the crisp values of the original AHP importance intensity scale in the range [1,9] regularly into three corresponding values $(l, m, u)$, as in the ta- 
Table 1

Linguistic scales for the intensity importance of the pair-wise comparisons matrix is made across the Fuzzy-AHP for managerial AmI

\begin{tabular}{|c|c|c|c|}
\hline $\begin{array}{l}\text { Linguistic scale } \\
\text { for the importance }\end{array}$ & Abbreviation & $\begin{array}{l}\text { Triangular } \\
\text { fuzzy } \\
\text { number }\end{array}$ & $\begin{array}{l}\text { Reciprocal } \\
\text { triangular } \\
\text { fuzzy number }\end{array}$ \\
\hline $\begin{array}{l}\text { Just equal/same of } \\
\text { i over } \mathrm{j}\end{array}$ & $\mathrm{Sm}$ & $(1,1,1)$ & $(1,1,1)$ \\
\hline Equal importance & ei & $(1,1,2)$ & $(1 / 2,1,1)$ \\
\hline $\begin{array}{l}\text { Between Equal } \\
\text { and weak } \\
\text { importance of i } \\
\text { over j }\end{array}$ & bew & $(1,2,3)$ & $(1 / 3,1 / 2,1)$ \\
\hline $\begin{array}{l}\text { Weak importance } \\
\text { of } \mathrm{i} \text { over } \mathrm{j}\end{array}$ & wi & $(2,3,4)$ & $(1 / 4,1 / 3,1 / 2)$ \\
\hline $\begin{array}{l}\text { Between weak } \\
\text { and Strong } \\
\text { importance of i } \\
\text { over j }\end{array}$ & bws & $(3,4,5)$ & $(1 / 5,1 / 4,1 / 3)$ \\
\hline $\begin{array}{l}\text { Strong importance } \\
\text { of } \mathrm{i} \text { over } \mathrm{j}\end{array}$ & si & $(4,5,6)$ & $(1 / 6,1 / 5,1 / 4)$ \\
\hline $\begin{array}{l}\text { Between strong } \\
\text { and demonstrated } \\
\text { importance of i } \\
\text { over j }\end{array}$ & bsd & $(5,6,7)$ & $(1 / 7,1 / 6,1 / 5)$ \\
\hline $\begin{array}{l}\text { Demonstrated } \\
\text { importance of } \mathrm{i} \\
\text { over } \mathrm{j}\end{array}$ & $\mathrm{di}$ & $(6,7,8)$ & $(1 / 8,1 / 7,1 / 6)$ \\
\hline $\begin{array}{l}\text { Between } \\
\text { demonstrated and } \\
\text { absolute } \\
\text { importance of } \mathrm{i} \\
\text { over } \mathrm{j}\end{array}$ & bda & $(7,8,9)$ & $(1 / 9,1 / 8,1 / 7)$ \\
\hline $\begin{array}{l}\text { Absolute } \\
\text { importance of } \mathrm{i} \\
\text { over } \mathrm{j}\end{array}$ & ai & $(8,9,9)$ & $(1 / 9,1 / 9,1 / 8)$ \\
\hline
\end{tabular}

ble. Then, Table 1 shows the fuzzy numbers and their reciprocals from the original AHP scale. The relative importance intensity of workplace performance criteria, decision support functions and AmI features will be assigned through either individual or multiple experts. Then, a fuzzy positive reciprocal matrix is constructed as follows:

$$
\tilde{A}=\left[\tilde{a}_{i j}\right]
$$

and the geometric mean of each row is computed $\tilde{a}_{i j}=\left(l_{i j}, m_{i j} u_{i j}\right)$ :

$$
\tilde{r}_{i}=\prod_{j=1}^{n} \tilde{a}_{i j}
$$

The normalized weight $\tilde{w}_{i}$ is determined using the following formula [25]:

$$
\tilde{w}_{i}=\left(\frac{l_{i}}{\sum_{i=1}^{n} u_{i}}, \frac{m_{i}}{\sum_{i=1}^{n} m_{i}}, \frac{u_{i}}{\sum_{i=1}^{n} l_{i}}\right)
$$

- Step 2: Consistency check: The expert decision maker must redo the ratios when the comparison matrix fails to pass the consistency test. The value of $\lambda_{\max }(C R)$ is computed based on the modal value of the resulting fuzzy numbers, through employing the original Saaty's procedure but using the operations on fuzzy numbers. Then, if $C R>0.1$, the fuzzy judgment matrix must be revised until reaching or being below the 0.1 consistency value.

- Step 3: Synthesizing: The computed priorities obtained for each element at a lower level of the hierarchy is first multiplied, respectively, to the computed weights of the elements at the higher level, and then, it is summed up over all of the elements of the higher level to obtain the final weight or priority for each element.

- Step 4: Ranking: Given the final weights of each element in the Fuzzy-AHP hierarchy expressed in a fuzzy number form, we now must defuzzify these fuzzy priorities to be able to rank each element (i.e., either AmI feature in the lowest level, decision support function in the third level, or a workplace performance measure at the second level) in a non-fuzzy form. This task could be accomplished through using the Best Non-fuzzy Performance BNP values defuzzification method from Chen et al. (2008) [26]. The defuzzified value for the triangular fuzzy performance score can be calculated simply, by using fuzzy number's centroid, as follows:

$$
B N P=C\left(\tilde{a}_{i j}\right)=\frac{l+m+u}{3}
$$

The above defuzzified value is used to rank the compared fuzzy numbers.

In the next section, we illustrate how the proposed hierarchical model can be computationally applied using Fuzzy-AHP on the assessment of the significance relationship between AmI technologies and workplace performance measures. Some example applications in the manufacturing workplace are used to illustrate how the proposed hierarchical model could be implemented. 


\section{Application of the generalized assessment model in a manufacturing workplace}

In this section, we refer to the first prominent application of AmI technologies in manufacturing, the Ambient Intelligence Laboratory AMILAB [15], and we consider it to be a case study of an example manufacturing workplace application for illustrating the applicability of our proposed model in the manufacturing workplaces; this concern has not been sufficiently tackled in the literature before. The manufacturing environment of the AMILAB [15] built at Teckniker (Spain) has a high speed NC milling machine (a machine used for cutting profiles within processed metal work pieces that has its cutting mill controlled through a computer numerical control program) and a 6-axis robot. The AMILAB case study is described in detail below.

Given the AMILAB case study, the four levels of the proposed hierarchical model are the following:

The AMI at the AMILAB is composed of the following technologies:

- Interface technologies (voice recognition systems, head-mounted display and data gloves)

- Location and tracking systems based on RFID tags and sensor networks

- Wearable and portable computing devices

- Multi-agent systems for tracking, interfacing and $\mathrm{NC}$ control

The intended AmI features at the AMILAB: The synthesized collection of AMI technologies is intended to realize the following AmI features:

- Context awareness (user preferences, users' current activities, user locations, user profiles and devices at hand, people and service availabilities)

- Learning and adaptability to the user's context

- Multimodal, naturally broadened interaction

- Ubiquity (computing and communication)

This arrangement means that these features have the highest priority and are desirable for being realized, but it does not mean that the other AmI features cannot be useful. However, the significance of the other features could also be investigated.

The functions/tasks at AMILAB: The manufacturing system at AMILAB, which has a high-speed NC milling machine and a 6-axis robot, should technically perform the following basic tasks when interacting with a worker:
- Accessing and interfacing with the high speed NC Machine

- Setting up the machine

- Inputting the NC parts program and various other tooling data

- Maintaining the NC machine

- Monitoring processing operations and their outcomes

- Controlling the 6-axis robot: controlling the loading and unloading tasks of the robot

The relevant manufacturing systems performance measures: The general performance measures were defined in [26], which can also be used to assess the performance at the AMILAB. They are as follows:

- Time measures: average batch processing time, average lead time, changeover time, cycle time, machine downtime, mean flow time, on-time delivery, setup time, tact time, and throughput time.

- Cost measures: overhead cost, scrap cost, setup cost, tooling cost, total quality cost, unit labor cost, unit manufacturing costs, unit material cost, and work in progress.

- Quality measures: average outgoing quality limit, incoming quality, MTTF, not right the first time, process capability index, return rate, rework \%, scrap \%, vendor quality rate, and warranty claim $\%$.

- Flexibility measures: component reusability, delivery flexibility, machine flexibility, number of different parts, process flexibility, process similarity, routing flexibility, supply chain flexibility, total system flexibility, and volume flexibility.

- Productivity measures: assembly line effectiveness, direct labor productivity, machine effectiveness, network effectiveness, overall equipment effectiveness, return on assets, stock turn, throughput efficiency, total productive maintenance, and value-added per employee.

\subsection{Application of Fuzzy-AHP for assessment of AmI technology impacts on the manufacturing workplace performance}

Here, we apply the Fuzzy-AHP model to the configured hierarchy of the generalized model of the manufacturing workplace. The Fuzzy-AHP is adequate and effective in understanding and analyzing the complex vague relationship among AmI technologies, features and manufacturing workplace functions and perfor- 


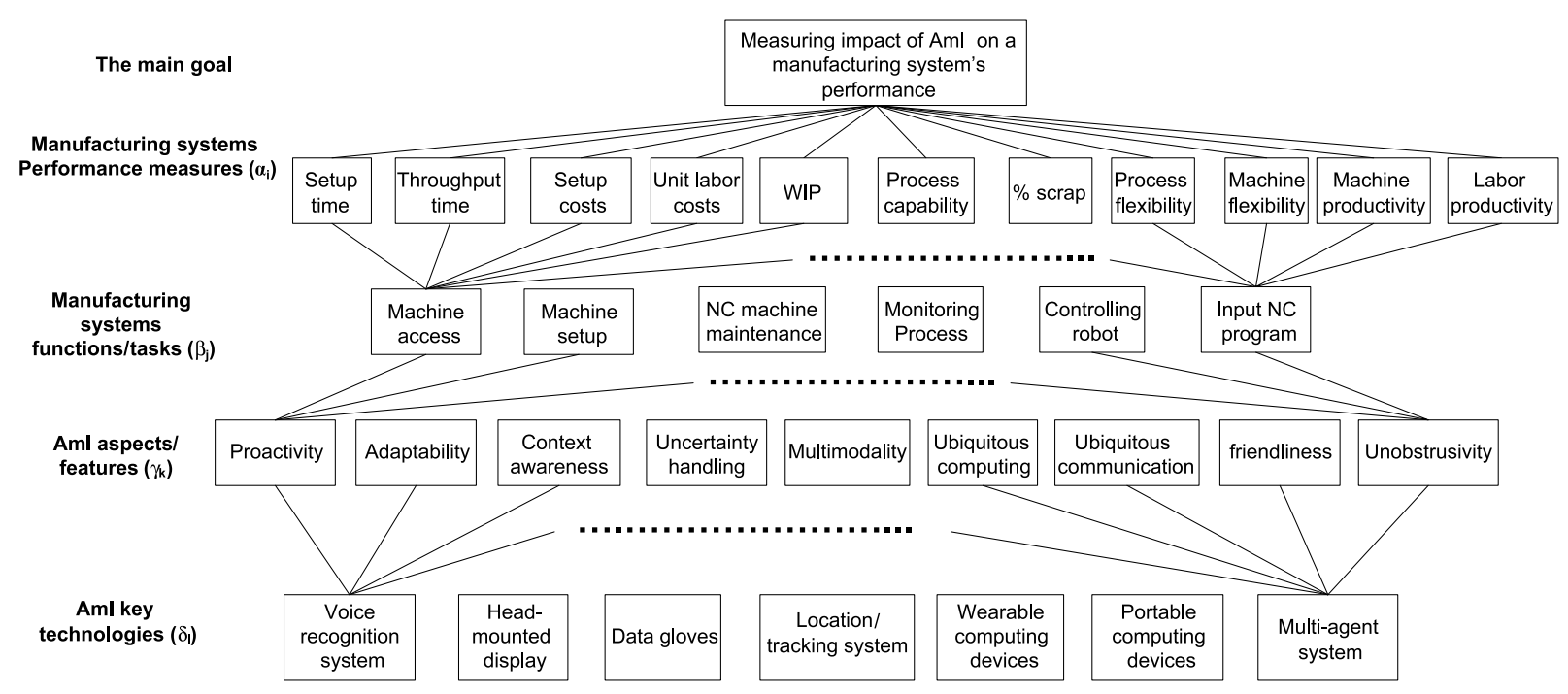

Fig. 6. The assessment hierarchy of the generalized model using Fuzzy-AHP.

mance measures. This capability will be achieved as follows:

- The structure of the Fuzzy-AHP hierarchy identically fits the developed conceptual hierarchy of the studied AmI technologies-performance relationship.

- The use of Fuzzy-AHP enables resorting to expert judgment that is an effective and adequate option for manipulating qualitative and vague values

- Fuzzy-AHP quantifies vague subjective concepts of various factors and quantities in the hierarchy through fuzzy numbers.

- The fuzzified AHP pair-wise intensity importances are considered and understood to be significant relationships of every quantity at one level with the other quantities at the next upper level.

- Exploiting and interpreting the significance values on both partial and overall relationship bases among and across the related levels.

- Computing synthesized priorities computed at the four levels of the hierarchy and computing the meaning of the final significance of the weights of the AmI technologies with respect to manufacturing workplace measures.

A fully detailed complete numerical example explanation of a computational procedure of Fuzzy-AHP is beyond the scope of this article, because it is a mainly systematic, number-crunching procedure with space limitations as well. However, a detailed step-by-step example can be found in [23].
Next, let us illustrate how the Fuzzy-AHP can be utilized to determine the importance of AmI technologies and features on the functions and the performance of the manufacturing workplace. We give a simple example about how the results of the evaluation of FuzzyAHP could be analyzed and can contribute to indicating the influence of the AmI key enabling technologies on the performance measures of the manufacturing workplace.

In the illustrative case study example that we show, we consider the AMILAB [15] and how the generalized hierarchy could be applied. The AMILAB experimental laboratory described in the previous section can now be taken as a case example to show how to quantify the impact of AmI features on the measures of performance of the lab. This task can now be accomplished through applying the described generalized hierarchy in Fig. 5. We can build the specific hierarchy for the relationship with the following:

- Level $\alpha$ : The manufacturing performance measures.

- Level $\beta$ : The basic manufacturing functions/tasks.

- Level $\gamma$ : The AmI features/attributes.

- Level $\delta$ : The AmI key enabling technologies.

The assessment hierarchy is shown below in the schematic in Fig. 7.

For convenience, let us use the following corresponding abbreviations for the Fuzzy-AHP levels in Fig. 7: 


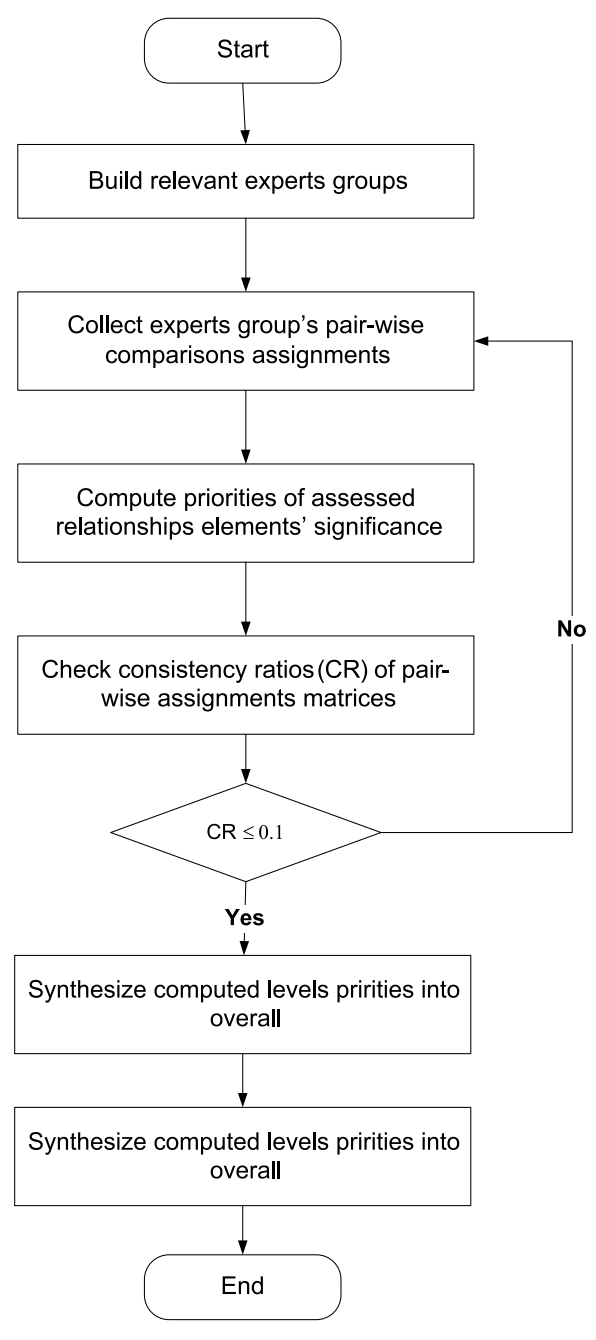

Fig. 7. The flowchart of the application of the Fuzzy-AHP procedure.

Level $\alpha$ : The manufacturing performance measures: Setup time $\left(\alpha_{1}\right)$, Throughput time $\left(\alpha_{2}\right)$, Setup cost $\left(\alpha_{3}\right)$, Unit labor costs $\left(\alpha_{4}\right)$, Work in Process (WIP) $\left(\alpha_{5}\right)$; Process capability $\left(\alpha_{6}\right)$; Percent scrap $\left(\alpha_{7}\right)$; Process flexibility $\left(\alpha_{8}\right)$; Machine flexibility $\left(\alpha_{9}\right)$; Machine productivity $\left(\alpha_{10}\right)$; and Labor productivity $\left(\alpha_{11}\right)$.

Let the final fuzzy priority of each performance measure with respect to the main goal be $\left(\breve{W} \alpha_{i}\right)(i=$ $1,2, \ldots, 11)$,

Level $\beta$ : The key manufacturing system function: Machine access $\left(\beta_{1}\right)$; Machine setup $\left(\beta_{2}\right)$; Data input $\left(\beta_{3}\right)$; NC machine maintenance $\left(\beta_{4}\right)$; Process monitoring $\left(\beta_{5}\right)$; and Controlling robot $\left(\beta_{6}\right)$.

Let the final priority of each be $\left(\check{W} \beta_{j}\right)(j=1,2$, $\ldots, 6)$, and the priority of $\beta_{j}$ with respect to each $i$ th performance measure is $\check{W} \beta_{j i}$.
Level $\gamma$ : The AmI attributes/features: Proactivity $\left(\gamma_{1}\right)$; Adaptability $\left(\gamma_{2}\right)$; Context awareness $\left(\gamma_{3}\right)$; Uncertainty handling $\left(\gamma_{4}\right)$; Multimodality $\left(\gamma_{5}\right)$; Ubiquitous computing $\left(\gamma_{6}\right)$; Ubiquitous communication $\left(\gamma_{7}\right)$; Friendliness $\left(\gamma_{8}\right)$; and Unobtructiveness $\left(\gamma_{9}\right)$.

Let the final fuzzy priority of $\gamma_{k}(k=1,2, \ldots, 9)$ with respect to each $j$ th system function be $\breve{W} \gamma_{k j}$.

Level $\delta$ : The AmI technologies: Voice recognition system $\left(\delta_{1}\right)$; Head-mounted display $\left(\delta_{2}\right)$; Data gloves $\left(\delta_{3}\right)$; Location/tracking system $\left(\delta_{4}\right)$; Wearable computing devices $\left(\delta_{5}\right)$; Portable computing devices $\left(\delta_{6}\right)$; and Multi-agent system $\left(\delta_{7}\right)$. Let the fuzzy priority of $\delta_{l}$, $(l=1,2, \ldots, 7)$ with respect to each $k$ th AmI feature be $\breve{W} \delta_{l k}$.

It should be noted that the priorities to be computed could be interpreted as either importances or significances for the relationships among the hierarchical elements.

The Fuzzy-AHP procedure starts with building a team of experts who have relevance, knowledge and expertise in AmI technologies and manufacturing systems. Several approaches could be used to build this team of experts. The recommended approach is to form a multidisciplinary team of experts, who could be selected based on three criteria: knowledge, relevance and experience. These experts should act through implementing the developed tools by the authors described in [23]. It is recommended that the experts' team meets together and participates together in making brain-storming sessions for addressing the anticipated impacts of AmI technologies and their embodied features on the functional activities and the associated performance measures. This arrangement will greatly help generating the sought interdisciplinary knowledge of AmI manufacturing that is needed to address such complex decision-making problems and to improve their assessment decision-making capability on the AmI impacts. The decision-makers should also become familiar with the designed Fuzzy-AHP scale. Then, entire evaluation decision-making tasks throughout the hierarchy could be shared among the individuals according to their desires, relevance, capabilities and background knowledge. Conflicts of opinion occur among experts, specifically when the impact of AmI features on system functions is resolved through the use of the geometric mean of the assigned values of the experts [28]. Similarly, multiple expert groups could be built to raise the reliability of the decision process outcome. Then, eventually the opinions of the participating groups of experts are synthesized 
Table 2

The pair-wise comparison matrix for the importance of the 11 manufacturing system performance measures

\begin{tabular}{|c|c|c|c|c|c|c|c|c|c|c|c|c|}
\hline & \multicolumn{11}{|c|}{ Manufacturing performance measures } & \multirow[t]{2}{*}{ Priority Fuzzy weights (Priority) $\check{W} \alpha_{i}$} \\
\hline & $\alpha_{1}$ & $\alpha_{2}$ & $\alpha_{3}$ & $\alpha_{4}$ & $\alpha_{5}$ & $\alpha_{6}$ & $\alpha_{7}$ & $\alpha_{8}$ & $\alpha_{9}$ & $\alpha_{10}$ & $\alpha_{11}$ & \\
\hline$\alpha_{1}$ & - & xbew & ai & $\mathrm{di}$ & wi & si & Bsd & bws & bda & ai & bew & $(0.13,0.21,0.33)$ \\
\hline$\alpha_{2}$ & & - & ai & bda & bws & bsd & di & si & ai & ai & wi & $(0.19,0.27,0.4)$ \\
\hline$\alpha_{3}$ & & & - & xbws & xbda & xbsd & xsi & xdi & xwi & xbew & xai & $(0.008,0.01,0.02)$ \\
\hline$\alpha_{4}$ & & & & - & $\mathrm{xsi}$ & xwi & xbew & xbws & bew & wi & xbsd & $(0.02,0.03,0.05)$ \\
\hline$\alpha_{5}$ & & & & & - & wi & bws & bew & bsd & di & xbew & $(0.07,0.11,0.19)$ \\
\hline$\alpha_{6}$ & & & & & & - & bew & xbew & bws & si & xbws & $(0.04,0.06,0.1)$ \\
\hline$\alpha_{7}$ & & & & & & & - & xwi & wi & bws & $\mathrm{xsi}$ & $(0.03,0.04,0.07)$ \\
\hline$\alpha_{8}$ & & & & & & & & - & si & bsd & xwi & $(0.05,0.08,0.13)$ \\
\hline$\alpha_{9}$ & & & & & & & & & - & bew & xdi & $(0.01,0.02,0.03)$ \\
\hline$\alpha_{10}$ & & & & & & & & & & - & xbda & $(0.01,0.02,0.03)$ \\
\hline$\alpha_{11}$ & & & & & & & & & & & - & $(0.1,0.16,0.25)$ \\
\hline
\end{tabular}

$C R=0.075 \leqslant 0.1$

or combined using the geometric mean, as described in [28].

The flowchart on Fig. 7 summarizes the Fuzzy-AHP assessment computational procedure for the AmI technologies impact.

Now, the Fuzzy-AHP procedure is ready to be applied. Using the scale in Table 1, the team of experts starts to compare the hierarchy elements level by level with respect to each factor at the next higher level. For simplicity and for the purpose of illustration and additionally because of the limitations in space, we consider the example opinion of one expert group and only the assessment with respect to the most important measure of performance of the manufacturing workplace, as will be indicated through the example computation. A sample of computations of a Fuzzy-AHP procedure are shown hereinafter.

Level $\alpha$ : Comparing the manufacturing performance measures (evaluating the performance measures with respect to the main hierarchical goal):

It is apparent from the table that the "Throughput", $\alpha_{2}$ performance measure has the highest crisp importance or priority when computed using the BNP formula (4), as follows:

$$
\begin{aligned}
B N P_{\alpha 2} & =\frac{u_{\alpha 2}+m_{\alpha 2}+l_{\alpha 2}}{3} \\
& =\frac{0.19+0.27+0.4}{3}=0.287
\end{aligned}
$$

A normalized crisp value is computed through summing the BNPs of all of the $\alpha$ 's and then re-dividing the values by the sum. The normalized value of the pri- ority of $\alpha_{2}$ is $W \alpha_{2}=0.25$. See the Appendix for a detailed computation of the crisp normalized priorities of the performance measures $\alpha_{\mathrm{j}}$ (Table 19).

Let us focus (for briefing and illustration purposes) on this performance measure and compute the significances or importances of the other elements in the hierarchy with respect to it.

Level $\beta$ : Comparing key manufacturing system functions: (evaluating key manufacturing functions with respect to the throughput $\left.\alpha_{2}\right)$ : Machine access $\left(\beta_{1}\right)$; Machine setup $\left(\beta_{2}\right)$; Data input $\left(\beta_{3}\right)$; NC machine maintenance $\left(\beta_{4}\right)$; Process monitoring $\left(\beta_{5}\right)$; and Controlling robot $\left(\beta_{6}\right)$. Table 3 shows the experts' assigned intensities for the compared manufacturing functions $\left(\check{W} \beta_{j 2}\right.$ ) and the computed priorities with respect to the $\alpha_{2}$ throughput measure.

Level $\gamma$ : The AmI attributes/features: Proactivity $\left(\gamma_{1}\right)$; Adaptability $\left(\gamma_{2}\right)$; Context awareness $\left(\gamma_{3}\right)$; Uncertainty handling $\left(\gamma_{4}\right)$; Multimodality $\left(\gamma_{5}\right)$; Ubiquitous computing $\left(\gamma_{6}\right)$; Ubiquitous communication $\left(\gamma_{7}\right)$; Friendliness $\left(\gamma_{8}\right)$; and Unobtructiveness $\left(\gamma_{9}\right)$.

Let the final priority of $\gamma_{k}(k=1,2, \ldots, 9)$ with respect to each $j$ th system function be $W \gamma_{k j}$.

The experts' assignments are given in the Appendix (Tables 4-9).

Level $\delta$ : The AmI technologies: Voice recognition system $\left(\delta_{1}\right)$; Head-mounted display $\left(\delta_{2}\right)$; Data gloves $\left(\delta_{3}\right)$; Location/tracking system $\left(\delta_{4}\right)$; Wearable computing devices $\left(\delta_{5}\right)$; Portable computing devices $\left(\delta_{6}\right)$; and Multi-agent system $\left(\delta_{7}\right)$. Let the priority of $\delta_{l}$, $(l=1,2, \ldots, 7)$ with respect to each $k$ th AmI feature be $W \delta_{l k}$. 
Table 3

The pair-wise comparison matrix for the significance of the 6 manufacturing system functions with respect to the Throughput performance measure

\begin{tabular}{|c|c|c|c|c|c|c|c|}
\hline \multirow[t]{2}{*}{$\alpha_{2}:$ Throughput time } & \multicolumn{6}{|c|}{ Manufacturing system key functions } & \multirow{2}{*}{ Fuzzy weights (Priority) $\check{W} \beta_{j}$} \\
\hline & $\overline{\beta_{1}}$ & $\beta_{2}$ & $\beta_{3}$ & $\beta_{4}$ & $\beta_{5}$ & $\beta_{6}$ & \\
\hline$\overline{\beta_{1}}$ & - & bew & xwi & bws & wi & xbew & $(0.09,0.16,0.3)$ \\
\hline$\beta_{2}$ & & - & xbws & wi & bew & xwi & $(0.06,0.0 .1,0.2)$ \\
\hline$\beta_{3}$ & & & - & bsd & si & bew & $(0.2,0.38,0.64)$ \\
\hline$\beta_{4}$ & & & & - & xbew & xsi & $(0.03,0.04,0.08)$ \\
\hline$\beta_{5}$ & & & & & - & xbws & $(0.04,0.06,0.12)$ \\
\hline$\underline{\beta_{6}}$ & & & & & & - & $(0.14,0.25,0.46)$ \\
\hline
\end{tabular}

Level $\gamma$ : The AmI attributes/features: Proactivity $\left(\gamma_{1}\right)$; Adaptability $\left(\gamma_{2}\right)$; Context awareness $\left(\gamma_{3}\right)$; Uncertainty handling $\left(\gamma_{4}\right)$; Multimodality $\left(\gamma_{5}\right)$; Ubiquitous computing $\left(\gamma_{6}\right)$; Ubiquitous communication $\left(\gamma_{7}\right)$; Friendliness $\left(\gamma_{8}\right)$; and Unobtructiveness $\left(\gamma_{9}\right)$.

Let the final priority of $\gamma_{k}(k=1,2, \ldots, 9)$ with respect to each $j$ th system function be $W \gamma_{k j}$.

The experts' assignments are given in the Appendix (Tables 4-9).

Level $\delta:$ The AmI technologies: Voice recognition system $\left(\delta_{1}\right)$; Head-mounted display $\left(\delta_{2}\right)$; Data gloves $\left(\delta_{3}\right)$; Location/tracking system $\left(\delta_{4}\right)$; Wearable computing devices $\left(\delta_{5}\right)$; Portable computing devices $\left(\delta_{6}\right)$; and Multi-agent system $\left(\delta_{7}\right)$. Let the priority of $\delta_{l}$, $(l=1,2, \ldots, 7)$ with respect to each $k$ th AmI feature be $W \delta_{l k}$.

The expert assignments are given in the Appendix (Tables 9-18). After applying the experts' pair-wise comparisons and given the computed final weight (priorities), a substantial amount of relationship information can be gained. The final overall priorities of the AmI technologies (for the purpose of illustration and considering only the most important performance measure $\alpha_{2}$, which is "Throughput time") can now be synthesized. This task can be accomplished through computing level-by-level the final priorities with respect to the upper performance measure levels, as described in $[2,23,28]$. The computations are as follows:

1. Synthesizing the final priorities of the AmI features w.r.t. the throughput performance measures:

$$
\begin{aligned}
\check{W} \gamma_{k}^{\alpha_{2}}= & \sum_{J=1}^{6} \check{W} \gamma_{k}^{\beta_{j}} * \check{W} \beta_{J}^{\alpha_{2}} \\
\check{W} \gamma_{1}^{\alpha_{2}}= & \check{W} \gamma_{1}^{\beta_{1}} * \check{W} \beta_{1}^{\alpha_{2}}+\check{W} \gamma_{1}^{\beta_{2}} * \check{W} \beta_{2}^{\alpha_{2}} \\
& +\check{W} \gamma_{1}^{\beta_{3}} * \check{W} \beta_{3}^{\alpha_{2}}+\check{W} \gamma_{1}^{\beta_{4}} * \check{W} \beta_{4}^{\alpha_{2}}
\end{aligned}
$$

$$
\begin{aligned}
& +\check{W} \gamma_{1}^{\beta_{5}} * \check{W} \beta_{5}^{\alpha_{2}}+\check{W} \gamma_{1}^{\beta_{6}} * \check{W} \beta_{6}^{\alpha_{2}} \\
= & (0.04,0.08,0.13) *(0.09,0.16,0.3) \\
& +(0.09,0.16,0.27) *(0.06,0.1,0.2) \\
& +(0.06,0.11,0.19) *(0.2,0.38,0.64) \\
& +(0.13,0.2,0.4) *(0.03,0.04,0.08) \\
& +(0.19,0.31,0.5) *(0.04,0.06,0.12) \\
& +(0.03,0.05,0.09) *(0.14,0.25,0.46) \\
\check{W} \gamma_{1}^{\alpha_{2}}= & (0.04,0.11,0.35)
\end{aligned}
$$

Then, $\check{W} \gamma_{2}^{\alpha_{2}}$ up to $\check{W} \gamma_{9}^{\alpha_{2}}$ are similarly computed as in Table 20 in the Appendix.

2. Synthesizing: final significance priorities of AmI technologies w.r.t. the "Throughput" performance measure $\alpha_{2}$ :

$$
\begin{aligned}
\check{W} \delta_{k}^{\alpha_{2}}= & \sum_{k=1}^{9} \check{W} \delta_{l}^{\gamma_{k}} * \check{W} \gamma_{k}^{\alpha_{2}} \\
\check{W} \delta_{1}^{\alpha_{2}}= & \check{W} \delta_{1}^{\gamma_{1}} * \check{W} \gamma_{1}^{\alpha_{2}}+\check{W} \delta_{1}^{\gamma_{2}} * \check{W} \gamma_{2}^{\alpha_{2}} \\
& +\check{W} \delta_{1}^{\gamma_{3}} * \check{W} \gamma_{3}^{\alpha_{2}}+\check{W} \delta_{1}^{\gamma_{4}} * \check{W} \gamma_{4}^{\alpha_{2}} \\
& +\check{W} \delta_{1}^{\gamma_{5}} * \check{W} \gamma_{5}^{\alpha_{2}}+\check{W} \delta_{6}^{\gamma_{6}} * \check{W} \gamma_{6}^{\alpha_{2}} \\
& +\check{W} \delta_{1}^{\gamma_{7}} * \check{W} \gamma_{7}^{\alpha_{2}}+\check{W} \delta_{1}^{\gamma_{8}} * \check{W} \gamma_{8}^{\alpha_{2}} \\
& +\check{W} \delta_{1}^{\gamma_{9}} * \check{W} \gamma_{9}^{\alpha_{2}} \\
= & (0.03,0.05,0.07) *(0.04,0.11,0.35) \\
& +(0.11,0.22,0.42) *(0.04,0.07,0.31) \\
& +(0.2,0.35,0.58) *(0.04,0.09,0.27) \\
& +(0.06,0.1,0.2) *(0.02,0.05,0.16) \\
& +(0.03,0.05,0.08) *(0.2,0.06,0.18)
\end{aligned}
$$




$$
\begin{aligned}
& +(0.09,0.16,0.3) *(0.05,0.13,0.4) \\
& +(0.14,0.25,0.46) *(0.06,0.19,0.56) \\
& +(0.06,0.1,0.19) *(0.04,0.12,0.38) \\
& +(0.02,0.03,0.06) *(0.06,0.16,0.48) \\
\check{W} \delta_{1}^{\alpha_{2}}= & (0.04,0.15,0.84)
\end{aligned}
$$

The remaining computations of the AmI technology priorities can be found in Table 21 in the Appendix.

The fuzzy weights can now be defuzzified using the BNP formula Eq. (4). The normalized crisp value of the significance priorities $W \beta, W \gamma$, and $W \delta$ are computed as in Tables 20-22. Then, the results indicate that the normalized BNPs with respect to the "Throughput" performance measures were found to be as follows:

$\beta$ level (Manufacturing system functions): The highest priority normalized BNP belongs to $\beta 3$, "CNC machine maintenance", which has a normalized $B N P_{\beta 3}=0.37$. This finding means that this function (when compared to other functions) is considered to be the most significant function for the improvement of the "Throughput" $\alpha_{2}$, a performance measure.

$\gamma$ level (AmI key features): The highest priority that normalized BNP belongs to is $\gamma_{7}$, "Ubiquitous Communication", which has a normalized $B N P_{\gamma 7}=0.18$. This finding means that this key AmI feature, when compared to other features, is considered to be the most significant for the improvement of the "Throughput" $\alpha_{2}$, a performance measure.

$\delta$ level (AmI technologies): The highest priority normalized BNP belongs to $\delta_{2}$, "Head-mounted display", which has a normalized $B N P_{\delta 2}=0.22$. This finding means that this key AmI technology, when compared to the other AmI technologies, is considered to be the most significant or has the highest impact on the improvement of the "Throughput" $\alpha_{2}$ performance measure.

The above results represent the final significance or importance priorities that were computed with respect to the most important computed performance measure, "Throughput". However, this case example could be extended to measure the significance priorities of the AmI technologies, AmI features and systems' function with respect to the other performance measures. Then, the values of all of the assessed elements in all of the levels can be synthesized to determine the overall significance priorities with respect to all of the perfor- mances measured through the same described synthesizing formulas, which is similar to Eqs (5) and (6).

The computed priorities of the pair-wise comparison results of this hierarchical assessment procedure are very beneficial, prior to designing the smart workplace and the utilization of AmI technologies in adding values to the measures of performance. These priority results of the assessments provide very rich information content about the significance and importance priorities between different elements in the hierarchy. These are the AmI technologies, AmI features, the key manufacturing function and, lastly, the performance measures. In this way, the computed priorities give insights about the significance and influence of AmI technologies or the following:

- The realized AmI features.

- Their significance to the key workplace functions.

- The final judgment yardstick; the performance measures of the workplace.

Over and above, the computed priorities among various elements, and with respect to other upper-level elements, provides valuable information on the significance, importance and influence relationships between elements at the same level and between those elements and other elements that are at successively higher levels. This information constitutes very valuable insight prior to designing the workplace and prior to adopting those AmI technologies as components that are specific to our application.

\section{Conclusions}

Ambient Intelligence (AmI) is a promising notion for improving the performance of manufacturing system environment functions. To consistently and justifiably implement AmI technologies in the manufacturing environment, it is crucial to study, understand and assess the impact of these newly emerging technologies on the performance of various actors and tasks in such an environment. However, due to subjectivity or intangibility, ill-structuredness and, consequently, inherent vagueness, it is difficult to accurately quantify and measure the impact of AmI technologies on the manufacturing environment performance. Generally speaking, such relationships between AmI technologies and features and the functional performance of the people and system are still an open question. Answering this question is valuable with respect to 
wisely and effectively exploiting AmI in useful directions.

This article intends to take a large step toward quantifying the influence of AmI features and attributes on the performance of managerial decision support. The article first logically structured and generalized the relationships among AmI technologies and the performance measures of any workplace. We then focused on the manufacturing environment as a typical complex workplace, which has not been sufficiently addressed in previous recent AmI applications literature. We then proposed a Fuzzy Analytical Hierarchical Process (Fuzzy-AHP) model as an adequately matching technique for measuring and quantifying and prioritizing the vague influence of various AmI technologies and features on the performance in the manufacturing environment workplace.

The use of Fuzzy-AHP offers the following advantages:

- The ability to use fuzzy numbers and linguistic values in expert judgments to address the assessment complexity and vagueness and to be simplified through pair-wise comparisons.

- The arrangement of the AmI technologies at the lowest level and the manufacturing performance measures at the upper-most level enables computing the final priority of each these AmI technologies with respect to the overall performance of the manufacturing system and computing the individual priorities as well for each performance criterion. This computation effectively contributes to answering the major question of this research.
- The use of the intermediate level of AmI features and manufacturing systems functions naturally to solve the problem and reduce the complexity of the studied relationship.

The proposed approach can be adequately used in the absence of more accurate techniques of assessment, in identifying the most important AmI technologies for each function and the performance measure of any workplace. It can also highlight the most important or crucial AmI features and functions for the overall performance of the workplace as a whole. In fact, the proposed approach provides very rich information on both the overall and the specific assessment basis of the evaluated factors and measures. Accordingly, based on this analysis, AmI key enabling technologies can be selected. Lastly, the proposed approach is practical and provides a good solution given the infeasibility of having optimum-yielding techniques that can quantify a non-homogenous mix of mainly qualitative or subjective factors and characteristics of quantitative performance measures.

\section{Acknowledgments}

This work was conducted within and related to the activities of the projects DEPIES - Decision Processes in Intelligent Environments and SOOW - Smart Offices and Other Workplaces, which were funded through the Czech Science Foundation, Czech Republic and the Post-Doc project of the CULS.

\section{Appendix A}

Table 4

The pair-wise comparisons matrix for the significance of the 9 AmI features with respect to the Machine access function

\begin{tabular}{|c|c|c|c|c|c|c|c|c|c|c|}
\hline \multirow[t]{2}{*}{$\beta_{1}:$ Machine access } & \multicolumn{10}{|c|}{ AmI's key features } \\
\hline & $\overline{\gamma_{1}}$ & $\gamma_{2}$ & $\gamma_{3}$ & $\gamma_{4}$ & $\gamma_{5}$ & $\gamma_{6}$ & $\gamma_{7}$ & $\gamma_{8}$ & $\gamma_{9}$ & $\begin{array}{l}\text { Fuzzy weights } \\
\text { (priority) } \breve{W} \gamma_{k}^{\beta_{1}}\end{array}$ \\
\hline$\overline{\gamma_{1}}$ & - & xwi & xsi & xbew & bew & xbws & si & bws & wi & $(0.04,0.08,0.13)$ \\
\hline$\gamma_{2}$ & & - & xwi & bew & bws & xbew & di & bsd & di & $(0.1,0.16,0.28)$ \\
\hline$\gamma_{3}$ & & & - & bws & bsd & bew & ai & bda & di & $(0.19,0.31,0.48)$ \\
\hline$\gamma_{4}$ & & & & - & wi & xwi & bsd & si & bws & $(0.08,0.14,0.25)$ \\
\hline$\gamma_{5}$ & & & & & - & xsi & bws & wi & bew & $(0.03,0.05,0.08)$ \\
\hline$\gamma_{6}$ & & & & & & - & bda & di & bsd & $(0.11,0.18,0.32)$ \\
\hline$\gamma_{7}$ & & & & & & & - & xbew & xwi & $(0.012,0.02,0.03)$ \\
\hline$\gamma_{8}$ & & & & & & & & - & xbew & $(0.02,0.025,0.04)$ \\
\hline$\gamma_{9}$ & & & & & & & & & - & $(0.02,0.03,0.06)$ \\
\hline
\end{tabular}


Table 5

The pair-wise comparisons matrix for the significance of the $9 \mathrm{AmI}$ features with respect to the Machine setup function

\begin{tabular}{|c|c|c|c|c|c|c|c|c|c|c|}
\hline \multirow[t]{2}{*}{$\beta_{2}:$ Machine setup } & \multicolumn{10}{|c|}{ AmI's key features } \\
\hline & $\gamma_{1}$ & $\gamma_{2}$ & $\gamma_{3}$ & $\gamma_{4}$ & $\gamma_{5}$ & $\gamma_{6}$ & $\gamma_{7}$ & $\gamma_{8}$ & $\gamma_{9}$ & $\begin{array}{l}\text { Fuzzy weights } \\
\text { (priority) } \breve{W} \gamma_{k}^{\beta_{2}}\end{array}$ \\
\hline$\gamma_{1}$ & - & xwi & xsi & bsd & bew & bws & xbew & di & si & $(0.09,0.16,0.27)$ \\
\hline$\gamma_{2}$ & & - & si & bda & bws & bsd & bew & ai & di & $(0.19,0.31,0.5)$ \\
\hline$\gamma_{3}$ & & & - & bws & xbew & bew & xbws & si & wi & $(0.04,0.07,0.13)$ \\
\hline$\gamma_{4}$ & & & & - & xsi & xwi & xdi & bew & xbew & $(0.02,0.025,0.04)$ \\
\hline$\gamma_{5}$ & & & & & - & wi & xwi & bsd & bws & $(0.06,0.11,0.19)$ \\
\hline$\gamma_{6}$ & & & & & & - & $\mathrm{xsi}$ & bws & bew & $(0.03,0.05,0.09)$ \\
\hline$\gamma_{7}$ & & & & & & & - & bda & bsd & $(0.13,0.2,0.4)$ \\
\hline$\gamma_{8}$ & & & & & & & & - & xwi & $(0.01,0.02,0.03)$ \\
\hline$\gamma_{9}$ & & & & & & & & & - & $(0.02,0.04,0.06)$ \\
\hline
\end{tabular}

Table 6

The pair-wise comparisons matrix for the significance of the $9 \mathrm{AmI}$ features with respect to the Data input function

\begin{tabular}{|c|c|c|c|c|c|c|c|c|c|c|}
\hline \multirow[t]{2}{*}{$\overline{\beta_{3}: \text { Data input }}$} & \multicolumn{10}{|c|}{ AmI's key features } \\
\hline & $\overline{\gamma_{1}}$ & $\gamma_{2}$ & $\gamma_{3}$ & $\gamma_{4}$ & $\gamma_{5}$ & $\gamma_{6}$ & $\gamma_{7}$ & $\gamma_{8}$ & $\gamma_{9}$ & $\begin{array}{l}\text { Fuzzy weights } \\
\text { (priority) } \breve{W} \gamma_{k}^{\beta_{3}}\end{array}$ \\
\hline$\gamma_{1}$ & - & bew & wi & bws & si & bsd & xbws & xwi & xbew & $(0.06,0.11,0.19)$ \\
\hline$\gamma_{2}$ & & - & bew & wi & bws & si & xsi & xbws & xwi & $(0.04,0.07,0.13)$ \\
\hline$\gamma_{3}$ & & & - & bew & wi & bws & xbsd & xsi & xbws & $(0.03,0.05,0.09)$ \\
\hline$\gamma_{4}$ & & & & - & bew & wi & xdi & xbsd & xsi & $(0.02,0.04,0.06)$ \\
\hline$\gamma_{5}$ & & & & & - & bew & xbda & xdi & xbsd & $(0.02,0.03,0.04)$ \\
\hline$\gamma_{6}$ & & & & & & - & xai & xbda & xdi & $(0.01,0.02,0.03)$ \\
\hline$\gamma_{7}$ & & & & & & & - & bew & wi & $(0.19,0.3,0.5)$ \\
\hline$\gamma_{8}$ & & & & & & & & - & bew & $(0.13,0.22,0.4)$ \\
\hline$\gamma_{9}$ & & & & & & & & & - & $(0.09,0.16,0.27)$ \\
\hline
\end{tabular}

$C R=0.0345 \leqslant 0.1(\mathrm{OK})$.

Table 7

The pair-wise comparisons matrix for the significance of the 9 AmI features with respect to the Process monitoring function

\begin{tabular}{|c|c|c|c|c|c|c|c|c|c|c|}
\hline \multirow[t]{2}{*}{$\beta_{4}:$ Process monitoring } & \multicolumn{10}{|c|}{ AmI's key features } \\
\hline & $\overline{\gamma_{1}}$ & $\gamma_{2}$ & $\gamma_{3}$ & $\gamma_{4}$ & $\gamma_{5}$ & $\gamma_{6}$ & $\gamma_{7}$ & $\gamma_{8}$ & $\gamma_{9}$ & $\begin{array}{l}\text { Fuzzy weights } \\
\text { (priority) } \check{W} \gamma_{k}^{\beta_{4}}\end{array}$ \\
\hline$\overline{\gamma_{1}}$ & - & si & bsd & bws & $\mathrm{di}$ & wi & bew & bda & xbew & $(0.13,0.2,0.4)$ \\
\hline$\gamma_{2}$ & & - & bew & xbew & wi & xwi & xbws & bws & xbsd & $(0.03,0.05,0.09)$ \\
\hline$\gamma_{3}$ & & & - & xwi & bew & xbws & xsi & wi & xdi & $(0.02,0.04,0.06)$ \\
\hline$\gamma_{4}$ & & & & - & bws & xbew & xwi & si & xsi & $(0.04,0.07,0.13)$ \\
\hline$\gamma_{5}$ & & & & & - & xsi & xbsd & bew & xbda & $(0.02,0.025,0.04)$ \\
\hline$\gamma_{6}$ & & & & & & - & xbew & bsd & xbws & $(0.06,0.1,0.19)$ \\
\hline$\gamma_{7}$ & & & & & & & - & di & xwi & $(0.09,0.16,0.27)$ \\
\hline$\gamma_{8}$ & & & & & & & & - & xai & $(0.01,0.02,0.03)$ \\
\hline$\gamma_{9}$ & & & & & & & & & - & $(0.19,0.31,0.5)$ \\
\hline
\end{tabular}


Table 8

The pair-wise comparisons matrix for the significance of the 9 AmI features with respect to the Process monitoring function

\begin{tabular}{|c|c|c|c|c|c|c|c|c|c|c|}
\hline \multirow[t]{2}{*}{$\beta_{5}:$ Process monitoring } & \multicolumn{10}{|c|}{ AmI's key features } \\
\hline & $l \gamma_{1}$ & $\gamma_{2}$ & $l \gamma_{3}$ & $\gamma_{4}$ & $l \gamma_{5}$ & $\gamma_{6}$ & $l \gamma_{7}$ & $\gamma_{8}$ & $l \gamma_{9}$ & $\begin{array}{l}\text { Fuzzy weights } \\
\text { (priority) } \breve{W} \gamma_{k}^{\beta_{5}}\end{array}$ \\
\hline$\gamma_{1}$ & - & di & bew & bda & wi & bws & ai & si & bsd & $(0.19,0.31,0.5)$ \\
\hline$\gamma_{2}$ & & - & xbsd & bew & xsi & xbws & wi & xwi & xbew & $(0.02,0.04,0.06)$ \\
\hline$\gamma_{3}$ & & & - & di & bew & wi & bda & bws & si & $(0.13,0.22,0.4)$ \\
\hline$\gamma_{4}$ & & & & - & xbsd & xsi & bew & xbws & xwi & $(0.02,0.025,0.04)$ \\
\hline$\gamma_{5}$ & & & & & - & bew & di & wi & bws & $(0.09,0.16,0.27)$ \\
\hline$\gamma_{6}$ & & & & & & - & bsd & bew & wi & $(0.06,0.1,0.19)$ \\
\hline$\gamma 7$ & & & & & & & - & $\mathrm{xsi}$ & xbws & $(0.01,0.02,0.03)$ \\
\hline$\gamma_{8}$ & & & & & & & & - & bew & $(0.04,0.07,0.13)$ \\
\hline$\gamma_{9}$ & & & & & & & & & - & $(0.03,0.05,0.09)$ \\
\hline
\end{tabular}

$C R=0.035 \leqslant 0.1(\mathrm{OK})$.

Table 9

The pair-wise comparisons matrix for the significance of the $9 \mathrm{AmI}$ features with respect to the Controlling robot function

\begin{tabular}{|c|c|c|c|c|c|c|c|c|c|c|}
\hline \multirow[t]{2}{*}{$\beta_{6}:$ Controlling robot } & \multicolumn{10}{|c|}{ AmI's key features } \\
\hline & $\overline{\gamma_{1}}$ & $\gamma_{2}$ & $\gamma_{3}$ & $\gamma_{4}$ & $\gamma_{5}$ & $\gamma_{6}$ & $\gamma 7$ & $\gamma_{8}$ & $\gamma_{9}$ & $\begin{array}{l}\text { Fuzzy weights } \\
\text { (priority) } \check{W} \gamma_{k}^{\beta_{6}}\end{array}$ \\
\hline$\gamma_{1}$ & - & bew & wi & bws & xbew & xbsd & xsi & xbws & xwi & $(0.03,0.05,0.09)$ \\
\hline$\gamma_{2}$ & & - & bew & wi & xwi & xdi & xbsd & xsi & xbws & $(0.02,0.04,0.06)$ \\
\hline$\gamma_{3}$ & & & - & bew & xbws & xbda & xdi & xbsd & xsi & $(0.02,0.03,0.04)$ \\
\hline$\gamma_{4}$ & & & & - & xsi & xai & xbda & xdi & xbsd & $(0.01,0.02,0.03)$ \\
\hline$\gamma_{5}$ & & & & & - & $\mathrm{xsi}$ & xbws & xwi & xbew & $(0.04,0.07,0.13)$ \\
\hline$\gamma_{6}$ & & & & & & - & bew & wi & bws & $(0.19,0.3,0.5)$ \\
\hline$\gamma_{7}$ & & & & & & & - & bew & wi & $(0.09,0.16,0.27)$ \\
\hline$\gamma_{8}$ & & & & & & & & - & bew & $(0.06,0.11,0.19)$ \\
\hline$\gamma_{9}$ & & & & & & & & & - & $(0.19,0.31,0.5)$ \\
\hline
\end{tabular}

$C R=0.0345 \leqslant 0.1(\mathrm{OK})$.

Table 10

The pair-wise comparisons matrix for the significance of the 7 AMILAB technologies with respect to the Proactivity AmI feature

\begin{tabular}{|c|c|c|c|c|c|c|c|c|}
\hline \multirow[t]{2}{*}{$\overline{\gamma_{1}: \text { Proactivity }}$} & \multicolumn{8}{|c|}{ AmI's key enabling technologies } \\
\hline & $\overline{\delta_{1}}$ & $\delta_{2}$ & $\delta_{3}$ & $\delta_{4}$ & $\delta_{5}$ & $\delta_{6}$ & $\delta_{7}$ & $\begin{array}{l}\text { Fuzzy weights } \\
\text { (priority) } \check{W} \delta_{l}^{\gamma_{1}}\end{array}$ \\
\hline$\overline{\delta_{1}}$ & - & xbsd & xwi & $\mathrm{xdi}$ & xwi & $\mathrm{sm}$ & wi & $\overline{(0.03,0.05,0.07)}$ \\
\hline$\delta_{2}$ & & - & wi & xsi & wi & si & di & $(0.15,0.22,0.31)$ \\
\hline$\delta_{3}$ & & & - & xbsd & $\mathrm{sm}$ & wi & si & $(0.073,0.11,0.15)$ \\
\hline$\delta_{4}$ & & & & - & bsd & di & bda & $(0.33,0.46,0.62)$ \\
\hline$\delta_{5}$ & & & & & - & wi & si & $(0.07,0.11,0.15)$ \\
\hline$\delta_{6}$ & & & & & & - & wi & $(0.03,0.05,0.07)$ \\
\hline$\delta_{7}$ & & & & & & & - & $(0.02,0.02,0.04)$ \\
\hline
\end{tabular}

$C R=0.057 \leqslant 0.1(\mathrm{OK})$. 
Table 11

The pair-wise comparisons matrix for the significance of the 7 AMILAB technologies with respect to the Adaptivity AmI feature

\begin{tabular}{|c|c|c|c|c|c|c|c|c|}
\hline \multirow[t]{2}{*}{$\gamma_{2}:$ Adaptivity } & \multicolumn{8}{|c|}{ AmI's key enabling technologies } \\
\hline & $\overline{\delta_{1}}$ & $\delta_{2}$ & $\delta_{3}$ & $\delta_{4}$ & $\delta_{5}$ & $\delta_{6}$ & $\delta_{7}$ & $\begin{array}{l}\text { Fuzzy weights } \\
\text { (priority) } \check{W}^{\gamma_{2}}\end{array}$ \\
\hline$\overline{\delta_{1}}$ & - & xbew & bws & wi & bew & wi & bew & $(0.11,0.22,0.42)$ \\
\hline$\delta_{2}$ & & - & si & bws & wi & bws & wi & $(0.18,0.33,0.59)$ \\
\hline$\delta_{3}$ & & & - & xbew & xwi & xbew & xwi & $(0.03,0.05,0.1)$ \\
\hline$\delta_{4}$ & & & & - & xbew & $\mathrm{sm}$ & xbew & $(0.04,0.07,0.15)$ \\
\hline$\delta_{5}$ & & & & & - & bew & $\mathrm{sm}$ & $(0.07,0.13,0.25)$ \\
\hline$\delta_{6}$ & & & & & & - & xbew & $(0.04,0.07,0.15)$ \\
\hline$\delta_{7}$ & & & & & & & - & $(0.07,0.13,0.25)$ \\
\hline
\end{tabular}

Table 12

The pair-wise comparisons matrix for the significance of the 7 AMILAB technologies with respect to the Context awareness AmI feature

\begin{tabular}{|c|c|c|c|c|c|c|c|c|}
\hline \multirow[t]{2}{*}{$\gamma_{3}:$ Context awareness } & \multicolumn{8}{|c|}{ AmI's key enabling technologies } \\
\hline & $\delta_{1}$ & $\delta_{2}$ & $\delta_{3}$ & $\delta_{4}$ & $\delta_{5}$ & $\delta_{6}$ & $\delta_{7}$ & $\begin{array}{l}\text { Fuzzy weights } \\
\text { (priority) } \check{W} \delta_{l}^{\gamma_{3}}\end{array}$ \\
\hline$\delta_{1}$ & - & bew & wi & bws & si & bsd & di & $(0.2,0.35,0.58)$ \\
\hline$\delta_{2}$ & & - & bew & wi & wi & si & di & $(0.14,0.24,0.43)$ \\
\hline$\delta_{3}$ & & & - & bew & wi & wi & si & $(0.09,0.16,0.3)$ \\
\hline$\delta_{4}$ & & & & - & bew & wi & bda & $(0.06,0.1,0.2)$ \\
\hline$\delta_{5}$ & & & & & - & bew & wi & $(0.04,0.07,0.13)$ \\
\hline$\delta_{6}$ & & & & & & - & bew & $(0.03,0.05,0.08)$ \\
\hline$\delta_{7}$ & & & & & & & - & $(0.02,0.03,0.06)$ \\
\hline
\end{tabular}

$C R=0.025 \leqslant 0.1(\mathrm{OK})$.

Table 13

The pair-wise comparisons matrix for the significance of the 7 AMILAB technologies with respect to the Uncertainty handling AmI feature

\begin{tabular}{|c|c|c|c|c|c|c|c|c|}
\hline \multirow[t]{2}{*}{$\gamma_{4}:$ Uncertainty handling } & \multicolumn{8}{|c|}{ AmI's key enabling technologies } \\
\hline & $\overline{\delta_{1}}$ & $\delta_{2}$ & $\delta_{3}$ & $\delta_{4}$ & $\delta_{5}$ & $\delta_{6}$ & $\delta_{7}$ & $\begin{array}{l}\text { Fuzzy weights } \\
\text { (priority) } \mathscr{W} \delta_{l}^{\gamma_{4}}\end{array}$ \\
\hline$\delta_{1}$ & - & bew & wi & bws & xbew & xwi & xbws & $(0.06,0.1,0.2)$ \\
\hline$\delta_{2}$ & & - & bew & wi & xwi & xbws & xsi & $(0.04,0.07,0.13)$ \\
\hline$\delta_{3}$ & & & - & bew & xbws & xsi & xbsd & $(0.03,0.045,0.08)$ \\
\hline$\delta_{4}$ & & & & - & xsi & xbsd & xdi & $(0.02,0.03,0.055)$ \\
\hline$\delta_{5}$ & & & & & - & xbew & xwi & $(0.09,0.16,0.3)$ \\
\hline$\delta_{6}$ & & & & & & - & xbew & $(0.13,0.24,0.43)$ \\
\hline$\delta_{7}$ & & & & & & & - & $(0.2,0.35,0.6)$ \\
\hline
\end{tabular}

$\begin{array}{ll}C R=0.025 \leqslant 0.1(\mathrm{OK}) . & \text { Table } 14\end{array}$

The pair-wise comparisons matrix for the significance of the 7 AMILAB technologies with respect to the Multimodality AmI feature

\begin{tabular}{|c|c|c|c|c|c|c|c|c|}
\hline \multirow[t]{2}{*}{$\gamma_{5}:$ Multimodality } & \multicolumn{8}{|c|}{ AmI's key enabling technologies } \\
\hline & $\delta_{1}$ & $\delta_{2}$ & $\delta_{3}$ & $\delta_{4}$ & $\delta_{5}$ & $\delta_{6}$ & $\delta_{7}$ & $\begin{array}{l}\text { Fuzzy weights } \\
\text { (priority) } W \delta_{l}^{\gamma_{5}}\end{array}$ \\
\hline$\delta_{1}$ & - & bew & xbsd & xsi & xbws & xwi & xbew & $(0.03,0.05,0.08)$ \\
\hline$\delta_{2}$ & & - & xdi & xbsd & xsi & xbws & xwi & $(0.02,0.03,0.06)$ \\
\hline$\delta_{3}$ & & & - & bew & wi & bws & si & $(0.2,0.35,0.6)$ \\
\hline$\delta_{4}$ & & & & - & bew & wi & bws & $(0.14,0.24,0.43)$ \\
\hline$\delta_{5}$ & & & & & - & bew & wi & $(0.09,0.16,0.3)$ \\
\hline$\delta_{6}$ & & & & & & - & bew & $(0.06,0.1,0.2)$ \\
\hline$\delta_{7}$ & & & & & & & - & $(0.04,0.07,0.13)$ \\
\hline
\end{tabular}

$C R=0.025 \leqslant 0.1(\mathrm{OK})$. 
Table 15

The pair-wise comparisons matrix for the significance of the 7 AMILAB technologies with respect to the Ubiquitous computing AmI feature

\begin{tabular}{|c|c|c|c|c|c|c|c|c|}
\hline \multirow[t]{2}{*}{$\gamma_{6}:$ Ubiquitous computing } & \multicolumn{8}{|c|}{ AmI's key enabling technologies } \\
\hline & $\overline{\delta_{1}}$ & $\delta_{2}$ & $\delta_{3}$ & $\delta_{4}$ & $\delta_{5}$ & $\delta_{6}$ & $\delta_{7}$ & $\begin{array}{l}\text { Fuzzy weights } \\
\text { (priority) } \breve{W} \delta_{l}^{\gamma_{6}}\end{array}$ \\
\hline$\overline{\delta_{1}}$ & - & bew & wi & bws & si & xbew & xwi & $(0.09,0.16,0.3)$ \\
\hline$\delta_{2}$ & & - & bew & wi & xsi & xwi & xbws & $(0.06,0.1,0.2)$ \\
\hline$\delta_{3}$ & & & - & bew & wi & xbws & $\mathrm{xsi}$ & $(0.2,0.35,0.6)$ \\
\hline$\delta_{4}$ & & & & - & bew & $\mathrm{xsi}$ & xbsd & $(0.04,0.07,0.13)$ \\
\hline$\delta_{5}$ & & & & & - & xbsd & xdi & $(0.02,0.03,0.06)$ \\
\hline$\delta_{6}$ & & & & & & - & xbew & $(0.13,0.24,0.43)$ \\
\hline$\delta_{7}$ & & & & & & & - & $(0.2,0.35,0.58)$ \\
\hline
\end{tabular}

\begin{tabular}{ll}
\hline$C R=0.025 \leqslant 0.1(\mathrm{OK})$. & Table 16
\end{tabular}

The pair-wise comparisons matrix for the significance of the 7 AMILAB technologies with respect to the Ubiquitous communication AmI feature

\begin{tabular}{|c|c|c|c|c|c|c|c|c|}
\hline \multirow[t]{2}{*}{$\gamma_{7}$ : Ubiquitous communication } & \multicolumn{8}{|c|}{ AmI's key enabling technologies } \\
\hline & $\delta_{1}$ & $\delta_{2}$ & $\delta_{3}$ & $\delta_{4}$ & $\delta_{5}$ & $\delta_{6}$ & $\delta_{7}$ & $\begin{array}{l}\text { Fuzzy weights } \\
\text { (priority) } \mathscr{W} \delta_{l}^{\gamma 7}\end{array}$ \\
\hline$\delta_{1}$ & - & xbew & wi & bws & si & bws & bsd & $(0.14,0.25,0.46)$ \\
\hline$\delta_{2}$ & & - & bew & wi & bws & si & bsd & $(0.16,0.3,0.54)$ \\
\hline$\delta_{3}$ & & & - & xbew & wi & bew & bws & $(0.06,0.12,0.25)$ \\
\hline$\delta_{4}$ & & & & - & bws & wi & si & $(0.09,0.17,0.32)$ \\
\hline$\delta_{5}$ & & & & & - & xbew & bew & $(0.03,0.05,0.09)$ \\
\hline$\delta_{6}$ & & & & & & - & wi & $(0.04,0.07,0.14)$ \\
\hline$\delta_{7}$ & & & & & & & - & $(0.02,0.03,0.06)$ \\
\hline
\end{tabular}

$C R=0.034 \leqslant 0.1(\mathrm{OK}) . \quad$ Table 17

The pair-wise comparisons matrix for the significance of the 7 AMILAB technologies with respect to the Friendliness AmI feature

\begin{tabular}{|c|c|c|c|c|c|c|c|c|}
\hline \multirow[t]{2}{*}{$\gamma_{8}:$ Friendliness } & \multicolumn{8}{|c|}{ AmI's key enabling technologies } \\
\hline & $\delta_{1}$ & $\delta_{2}$ & $\delta_{3}$ & $\delta_{4}$ & $\delta_{5}$ & $\delta_{6}$ & $\delta_{7}$ & $\begin{array}{l}\text { Fuzzy weights } \\
\text { (priority) } \mathscr{W}_{l}^{\gamma_{l}^{\gamma}}\end{array}$ \\
\hline$\delta_{1}$ & - & bew & wi & bws & xbws & xwi & xbew & $(0.06,0.1,0.19)$ \\
\hline$\delta_{2}$ & & - & bew & wi & xsi & xbws & xwi & $(0.04,0.07,0.13)$ \\
\hline$\delta_{3}$ & & & - & bew & xbsd & xsi & xbws & $(0.03,0.05,0.08)$ \\
\hline$\delta_{4}$ & & & & - & xdi & xbsd & $\mathrm{xsi}$ & $(0.02,0.03,0.06)$ \\
\hline$\delta_{5}$ & & & & & - & bew & wi & $(0.2,0.4,0.6)$ \\
\hline$\delta_{6}$ & & & & & & - & bew & $(0.14,0.24,0.43)$ \\
\hline$\delta_{7}$ & & & & & & & - & $(0.09,0.16,0.3)$ \\
\hline
\end{tabular}

$C R=0.025 \leqslant 0.1(\mathrm{OK})$ Table 18

The pair-wise comparisons matrix for the significance of the 7 AMILAB technologies with respect to the Unobtrusivity AmI feature

\begin{tabular}{|c|c|c|c|c|c|c|c|c|}
\hline \multirow[t]{2}{*}{$\gamma_{9}:$ Unobtructiveness } & \multicolumn{8}{|c|}{ AmI's key enabling technologies } \\
\hline & $\delta_{1}$ & $\delta_{2}$ & $\delta_{3}$ & $\delta_{4}$ & $\delta_{5}$ & $\delta_{6}$ & $\delta_{7}$ & $\begin{array}{l}\text { Fuzzy weights } \\
\text { (priority) } \tilde{W} \delta_{l}^{\gamma_{9}}\end{array}$ \\
\hline$\overline{\delta_{1}}$ & - & $\mathrm{xbsd}$ & xbws & $\mathrm{xsi}$ & xdi & xbew & xwi & $(0.02,0.03,0.06)$ \\
\hline$\delta_{2}$ & & - & wi & bew & xbew & si & bws & $(0.14,0.24,0.43)$ \\
\hline$\delta_{3}$ & & & - & xbew & xbws & wi & bew & $(0.06,0.1,0.2)$ \\
\hline$\delta_{4}$ & & & & - & xwi & bws & wi & $(0.09,0.16,0.3)$ \\
\hline$\delta_{5}$ & & & & & - & bsd & si & $(0.2,0.35,0.58)$ \\
\hline$\delta_{6}$ & & & & & & - & xbew & $(0.03,0.0 .05,0.08)$ \\
\hline$\delta_{7}$ & & & & & & & - & $(0.04,0.07,0.13)$ \\
\hline
\end{tabular}


Table 19

Final synthesized crisp priorities for the AMILAB manufacturing performance measures

\begin{tabular}{|c|c|c|c|c|c|c|}
\hline$\alpha_{i}$ & $\alpha_{1}$ & $\alpha_{2}$ & $\alpha_{3}$ & $\alpha_{4}$ & $1 \alpha_{5}$ & $\alpha_{6}$ \\
\hline$\check{W} \alpha_{i}$ & $(0.13,0.21,0.33)$ & $(0.19,0.27,0.4)$ & $(0.008,0.01,0.2)$ & $(0.02,0.03,0.05)$ & $(0.07,0.11,0.19)$ & $(0.04,0.06,0.1)$ \\
\hline $\begin{array}{l}\text { Normalized Crisp } \\
\text { Priority } W \alpha_{i} \\
\end{array}$ & 0.19 & $0.25^{*}$ & 0.06 & 0.03 & 10.1 & 0.17 \\
\hline$\gamma_{k}$ & $\alpha_{7}$ & $\alpha_{8}$ & $\alpha_{9}$ & $\alpha_{10}$ & $\alpha_{11}$ & \\
\hline$\breve{W} \alpha_{i}$ & $(0.03,0.04,0.07)$ & $(0.05,0.08,0.13)$ & $(0.01,0.02,0.03)$ & $(0.01,0.02,0.03)$ & $(0.1,0.16,0.25)$ & \\
\hline $\begin{array}{l}\text { Normalized Crisp } \\
\text { Priority } W \alpha_{i}\end{array}$ & 0.04 & 0.08 & 0.02 & 0.02 & 0.15 & \\
\hline
\end{tabular}

Table 20

Final synthesized fuzzy priorities for the system functions w.r.t. the $\alpha_{2}$ "throughput" performance measure

\begin{tabular}{|c|c|c|c|c|c|c|}
\hline$\overline{\beta_{j}}$ & $\beta_{1}$ & $\beta_{2}$ & $\beta_{3}$ & $\beta_{4}$ & $\beta_{5}$ & $\beta_{6}$ \\
\hline$\check{W} \beta_{j}^{\alpha_{2}}$ & $(0.09,0.16,0.3)$ & $(0.06,0.1,0.2)$ & $(0.2,0.38,0.64)$ & $(0.03,0.04,0.08)$ & $(0.04,0.06,0.12)$ & $(0.14,0.25,0.46)$ \\
\hline $\begin{array}{l}\text { Normalized Crisp } \\
\text { Priority } W \beta_{j}^{\alpha_{2}}\end{array}$ & 0.16 & 0.11 & $0.37^{*}$ & 0.05 & 0.07 & 0.26 \\
\hline
\end{tabular}
* BNP

Table 21

Final synthesized fuzzy priorities for the AmI technologies w.r.t. the $\alpha_{2}$ "throughput" performance measure

\begin{tabular}{|c|c|c|c|c|c|}
\hline$\gamma_{k}$ & $\gamma_{1}$ & $\gamma_{2}$ & $\gamma_{3}$ & $\gamma_{4}$ & $\gamma_{5}$ \\
\hline$\check{W} \gamma_{k}^{\alpha_{2}}$ & $(0.04,0.11,0.35)$ & $(0.04,0.07,0.31)$ & $(0.04,0.09,0.27)$ & $(0.02,0.05,0.16)$ & $(0.2,0.06,0.18)$ \\
\hline $\begin{array}{l}\text { Normalized Crisp } \\
\text { Priority } W \gamma_{k}^{\alpha_{2}}\end{array}$ & 0.12 & 0.095 & 0.09 & 0.05 & 0.06 \\
\hline $\begin{array}{l}\gamma_{k} \\
\check{W} \gamma_{k}^{\alpha_{2}}\end{array}$ & $\begin{array}{c}\gamma_{6} \\
(0.05,0.13,0.4)\end{array}$ & $\begin{array}{c}\gamma_{7} \\
(0.06,0.19,0.56)\end{array}$ & $\begin{array}{c}\gamma_{8} \\
(0.04,0.12,0.38)\end{array}$ & $\begin{array}{c}\gamma_{9} \\
(0.06,0.16,0.48)\end{array}$ & \\
\hline $\begin{array}{l}\text { Normalized Crisp } \\
\text { Priority } W \gamma_{k}^{\alpha_{2}}\end{array}$ & 0.13 & $0.18^{*}$ & 0.12 & 0.16 & \\
\hline
\end{tabular}

Table 22

Final synthesized fuzzy priorities for the AmI technologies w.r.t. the manufacturing performance measures

\begin{tabular}{|c|c|c|c|c|c|}
\hline$\delta_{l}$ & $\delta_{1}$ & $\delta_{2}$ & $\delta_{3}$ & $\delta_{4}$ & $\delta_{5}$ \\
\hline $\bar{W} \delta_{l}^{\alpha_{2}}$ & $(0.04,0.15,0.84)$ & $(0.05,0.22,1.24)$ & $(0.07,0.14,0.79)$ & $(0.05,0.11,0.63)$ & $(0.043,0.12,0.64)$ \\
\hline $\begin{array}{l}\text { Normalized Crisp } \\
\text { Priority } W \delta_{l}^{\alpha_{2}}\end{array}$ & 0.15 & $0.22^{*}$ & 0.15 & 0.12 & 0.12 \\
\hline$\delta_{l}$ & $\delta_{6}$ & $\delta_{7}$ & & & \\
\hline$\check{W} \delta_{l}^{\alpha_{2}}$ & $(0.035,0.11,0.65)$ & $(0.03,0.12,0.67)$ & & & \\
\hline $\begin{array}{l}\text { Normalized Crisp } \\
\text { Priority } W \delta_{l}^{\alpha_{2}}\end{array}$ & 0.12 & 0.12 & & & \\
\hline
\end{tabular}

${ }^{*} B N P$ 


\section{Appendix B}

\section{Fuzzy sets and fuzzy numbers}

Fuzzy set theory provides a framework for handling uncertainties. Zadeh (1965) [30] initiated fuzzy set theory. In a non-fuzzy set, every object is either a member of the set or is not a member of the set, but in fuzzy sets, every object is, to some extent, a member of a set, and to some extent, a member of another set [31]. Thus, unlike crisp sets, membership is a continuous concept in fuzzy sets. Fuzzy set theory is used in support of linguistic variables, and the ambiguity in a problem and is widely applicable in information gathering, modeling, analysis, optimization, control, decision making and supervision.

Fuzzy quantities such as fuzzy sets are usually used to express ambiguous quantities. There are two common types of fuzzy quantities (with respect to their membership function): the Triangular fuzzy quantity (i.e., the fuzzy quantity with a triangular membership function) and the Trapezoidal fuzzy quantity (i.e., the fuzzy quantity with a trapezoidal membership function). In the fuzzy set literature, we can often see concepts such as fuzzy number and fuzzy interval. Fuzzy number represents a special type of fuzzy quantity that has a membership function that reaches a value of 1 at only one point and is below 1 elsewhere. It follows from the definition that the triangular fuzzy quantity is a fuzzy number. A fuzzy quantity is referred to as a fuzzy interval if the membership function reaches the value of 1 in a certain interval and is below 1 outside of this interval. A Trapezoidal fuzzy quantity is, thus, by definition, a fuzzy interval.

A fuzzy number $\tilde{A}$ expresses the meaning 'about A'. The triangular membership function of a fuzzy number can be expressed by a trinity $(l, m, u)$, i.e., lower $(l)$, modal $(m)$, and upper $(u)$ values of a triangle base. A fuzzy number is defined as follows:

Definition. A fuzzy number $X$ on $R$ is defined to be a fuzzy number if its membership function $\mu_{x}: R \rightarrow$ $[0,1]$ is equal to:

$$
\mu_{x}= \begin{cases}\frac{1}{m-l} x-\frac{l}{m-l}, & x \in[l, m] \\ \frac{1}{m-u} x-\frac{u}{m-u}, & x \in[m, u] \\ 0 & \text { otherwise }\end{cases}
$$

where $l<m<u$. A fuzzy number, as expressed by Eq. (B.1), is denoted by $(l, m, u)$.

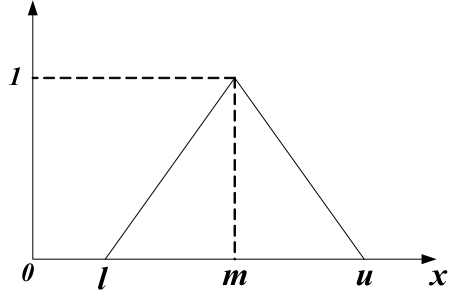

Fig. B.1. The membership of a fuzzy triangular number.

A fuzzy membership function and the definition of a fuzzy number are shown in Fig. B.1.

While a fuzzy number has a triangular membership function that is expressed by three numerical values $(l, m, u)$, in the case of a fuzzy interval, the fuzziness is expressed through four values $(a, b, c, d)$ (determining the shape of the trapezoidal membership function), with $b$ and $c$ having equal and maximum membership. In fact, a trapezoidal membership function is used when there is a range between the values $b$ and $c$, in which there is the highest degree of membership (equal to 1$)$.

Some basic relevant operations on fuzzy numbers that were developed and used in [24], [25] are defined as follows. For any two fuzzy numbers: $\tilde{A}=$ $\left(a_{1}, a_{2}, a_{3}\right), \tilde{B}=\left(b_{1}, b_{2}, b_{3}\right)$ :

$$
\begin{aligned}
& \tilde{A} \oplus \tilde{B} \\
& \quad=\left(a_{1}+b_{1}, a_{2}+b_{2}, a_{3}+b_{3}\right) \text { for addition } \\
& \tilde{A} \otimes \tilde{B} \\
& \quad=\left(a_{1} * b_{1}, a_{2} * b_{2}, a_{3} * b_{3}\right) \text { for multiplication } \\
& \tilde{A} / \tilde{B}=\left(a_{1} / b_{3}, a_{2} / b_{2}, a_{3} * b_{1}\right) \text { for division } \\
& 1 / \tilde{A}=\left(1 / a_{3}, 1 / a_{2}, 1 / a_{1}\right) \text { for reciprocal } \\
& (\tilde{A})^{n}=\left(a_{1}^{n}, a_{2}^{n}, a_{3}^{n}\right) \text { for power. }
\end{aligned}
$$

\section{References}

[1] ISTAG, IST Advisory Group, Advisory Group to the European Community's Information Society Technology Program, 2005, http://www.cordis.lu/ist/istag.htmi.

[2] D.J. Cook, J.C. Augusto and V.R. Jakkula, Ambient intelligence: Technologies, applications, and opportunities, Pervasive and Mobile Computing 5 (2009), 277-298.

[3] J. Augusto, Ambient intelligence: The confluence of pervasive computing and artificial intelligence, in: Intelligent Computing Everywhere, A. Schuster, ed., Springer, 2007, pp. 213-234.

[4] G. Riva, F. Vatalaro, F. Davide and M. Alcaniz, Ambient Intelligence. The Evolution of Technology, Comm. and Cognition Towards the Future of Human - Computer Interaction, IOS Press, Amsterdam, 2005, pp. 1-320. 
[5] E. Serrano and J. Botia, Validating ambient intelligence based ubiquitous computing systems by means of artificial societies, Information Sciences 222 (2013), 3-24.

[6] X. Hu, W. Du and B. Spencer, A multi-agent framework for Ambient systems development, Procedia Computer Science $\mathbf{5}$ (2011), 82-89.

[7] D.I. Tapia, J.A. Fraile, S. Rodríguez, R.S. Alonso and J.M. Corchado, Integrating hardware agents into an enhanced multi-agent architecture for Ambient Intelligence systems, Information Sciences 222 (2013), 47-65.

[8] M.J. Santofimia, S.E. Fahlman, X. del Toro, F. Moya and J.C. Lopez, A semantic model for actions and events in Ambient Intelligence, Engineering Applications of Artificial Intelligence 24(8) (2011), 1432-1445.

[9] T. Bosse, F. Both, C. Gerritsen, M. Hoogendoorn and Jan Treur, Methods for model-based reasoning within agentbased Ambient Intelligence applications, Knowledge-Based Systems 27 (2012), 190-210.

[10] N. Dipsis and K. Stathis, Ubiquitous agents for Ambient ecologies, Pervasive and Mobile Computing 8(4) (2012), 562-574.

[11] K. Vila, J.-N. Maźon and A. Ferŕandez, Model-driven adaptation of question answering systems for Ambient Intelligence by integrating restricted-domain knowledge, Procedia Computer Science 4 (2011), 1650-1659.

[12] A. De Paola, S. Gaglio, G. Lo Re and M. Ortolani, Sensorgk: A testbed for designing and experimenting with WSN-based Ambient Intelligence applications, Pervasive and Mobile Computing 8(3) (2012), 448-466.

[13] G. Kovács, S. Kopácsi, G. Haidegger and R. Michelini, Ambient Intelligence in product life-cycle management, Engineering Applications of Artificial Intelligence 19 (2006), 953-965.

[14] S. Kopácsi, G. Kovács, A. Anufriev and R. Michelini, Ambient intelligence as enabling technology for modern business paradigms, Robotics and Computer-Integrated Manufacturing 23(2) (2007), 242-256.

[15] I. Maurtua, M.A. Pérez, L. Susperregi, C. Tubio and A. Ibarguren, in: Ambient Intelligence in Manufacturing, Intelligent Production Machines and Systems, 2nd I*PROMS International Virtual Conference, July 2006, pp. 535-540.

[16] F. Doctor, H. Hagras and V. Callaghan, A type-2 fuzzy embedded agent to realize Ambient Intelligence in ubiquitous computing environments, Information Sciences 171(4) (2005), 309-334.

[17] G. Acampora and V. Loia, A proposal of ubiquitous fuzzy computing for Ambient Intelligence, Information Sciences 178(3) (2008), 631-646.
[18] I. Vrana and S. Aly, Conceptual models for managerial Ambient Intelligence, in: Ambient Intelligence forum, Hradec Kralove, Sept, 2009, Czech Republic, Ambient Intelligence Perspectives II, IOS Press, 2009, pp. 53-63.

[19] S. Aly and I. Vrana, Objective measurement of Ambient Intelligence influence on decision support in smart offices, in: Proc. of the 11th Smart Offices and Other Workplaces (SOOW'11) Workshop, The 7th International Conference on Intelligent Environments, 25-28 July, Nottingham, United Kingdom, 2011.

[20] J.-Y. Hong, E.-H. Suh and S.-J. Kim, Context-aware systems: A literature review and classification, Expert Systems with Applications 36(4) (2008), 8509-8522.

[21] J.C. Augusto, Ambient Intelligence: Basic concepts and applications, in: ICSOFT 2006, J. Filipe, B. Shishkov and M. Helfert, eds, CCIS, Vol. 10, Springer-Verlag, Berlin, Heidelberg, 2008, pp. 16-26.

[22] T.L. Saaty, The Analytic Hierarchy Process, McGraw-Hill, NY, 1980.

[23] S. Aly and I. Vrana, Evaluating the knowledge, relevance and experience of expert decision makers utilizing the Fuzzy-AHP, Agriculture Economics - Czech Republic 54(11) (2008), 529535.

[24] P.J.M. Laarhoven and W. Pedrycz, A fuzzy extension of Saaty's priority theory, Fuzzy Sets and Systems 11 (1983), 229-241.

[25] M. Dagdeviren and I. Yuksel, Developing a fuzzy analytic hierarchy process (AHP) model for behavior-based safety management, Information Sciences 178(6) (2008), 1717-1733.

[26] M.F. Chen, G.H. Tzeng and C.G. Ding, Combining fuzzy AHP with MDS in identifying the preference similarity of alternatives, Applied Soft Computing 8(1) (2008), 110-117.

[27] K.K.B. Hon, Performance and evaluation of manufacturing systems (keynote paper), Annals of CIRP 52(2) (2005), 675690.

[28] S. Aly, Integrating multiple fuzzy expert systems under varying requirements, $\mathrm{PhD}$ thesis, Czech University of Life Science in Prague, Prague, Czech Republic, 2006.

[29] Y.-C. Choua, C.-C. Sunb and H.-Y. Yenc, Evaluating the criteria for human resource for science and technology (HRST) based on an integrated fuzzy AHP and fuzzy DEMATEL approach, Applied Soft Computing 12 (2012), 64-71.

[30] L.A. Zadeh, Fuzzy sets, Information and Control 8 (1965), 338-353.

[31] M. Fasanghari, Knowledge management (intellectual capital) assessment using fuzzy expert system, in: Knowledge Management, P. Virtanen and N. Helander, eds, In-The, Croatia, 2010, pp. 15-24. 